\title{
On Cartesian products of signed graphs
}

\author{
Dimitri Lajou ${ }^{\mathrm{a}}$ \\ ${ }^{a}$ Univ. Bordeaux, Bordeaux INP, CNRS, LaBRI, UMR5800, F-33400 Talence, France. \\ email: dimitri.lajou@labri.fr
}

\begin{abstract}
In this paper, we study the Cartesian product of signed graphs as defined by Germina, Hameed and Zaslavsky (2011). Here we focus on its algebraic properties and look at the chromatic number of some Cartesian products. One of our main results is the unicity of the prime factor decomposition of signed graphs. This leads us to present an algorithm to compute this decomposition in linear time based on a decomposition algorithm for oriented graphs by Imrich and Peterin (2018). We also study the chromatic number of a signed graph, that is the minimum order of a signed graph to which the input signed graph admits a homomorphism, of graphs with underlying graph of the form $P_{n} \square P_{m}$, of Cartesian products of signed paths, of Cartesian products of signed complete graphs and of Cartesian products of signed cycles.
\end{abstract}

\section{Introduction}

Signed graphs were introduced by Harary in [8]. In 2005, Guenin introduced the notion of homomorphism of signed graphs, which was later studied by Naserasr, Rollová and Sopena [13]. This gave rise to a notion of chromatic number $\chi_{s}(G, \sigma)$ of a signed graph $(G, \sigma)$ defined as the smallest order of a signed graph $(H, \pi)$ to which $(G, \sigma)$ admits a homomorphism.

In this paper, we are interested in the study of Cartesian products of signed graphs, defined by Germina, Hameed and Zaslavsky in [7]. They mainly study the spectral properties of the Cartesian product. In this paper, we present algebraic properties of the Cartesian product and study the chromatic number of some Cartesian products of signed graphs.

The Cartesian product of two ordinary graphs $G$ and $H$, noted $G \square H$, has been extensively studied. In 1957, Sabidussi [14] showed that $\chi(G \square H)=\max (\chi(G), \chi(H))$ where $\chi(G)$ is the chromatic number of the graph $G$. Another notable article on the subject by Sabidussi [15] shows that every connected graph $G$ admits a unique prime decomposition, i.e. there is a unique way to write a graph $G$ as a product of some graphs up to isomorphism of the factors. This result was also independently discovered by Vizing in [16]. Another algebraic property, the cancellation property, which states that if $A \square B=A \square C$, then $B=C$, was proved by Imrich and Klavžar [10] using a technique of Fernández, Leighton and López-Presa [6]. On the complexity side, the main question associated with the Cartesian product is to decompose a graph with the best possible complexity. The complexity of this problem has been improved successively in 
$[5,17,4,1]$ to finally reach an optimal complexity of $O(m)$ in [11] where $m$ is the number of edges of the graph.

Our study of the Cartesian product of signed graphs is divided in several sections. First in section 2, we present general definitions of graph theory and set our notation. In section 3, we present some useful results on signed graphs and on the Cartesian product of ordinary graphs. In section 4, we present the definition of the Cartesian product of signed graphs and give some first properties and easy consequences of the definition. We also prove the prime decomposition theorem for signed graphs and give an algorithm to decompose a Cartesian product of signed graphs into its factors. We study the chromatic number of Cartesian products of signed complete graphs in section 5 and products of cycles in section 6 . Finally we present some concluding remarks in section 7 .

\section{Definitions and notation}

All graphs we consider are undirected, simple and loopless. For classical graph definitions, we refer the reader to the book Graph Theory by Bondy and Murty [2].

Two vertices $u$ and $v$ of a graph $G$ are said to be adjacent when $u v$ is an edge of $G$. An edge $u v$ is incident with a vertex $w$ if and only if $w$ is one of $u$ or $v$. The neighborhood $N_{G}(u)$ of a vertex $u$ in the graph $G$ is the set of vertices adjacent to $u$ in $G$. When the context is clear, we note $N(u)$ for the neighborhood of $u$ in $G$. The order of $G$ is $|V(G)|$ and its size is $|E(G)|$ where $|X|$ is the cardinal of a set $X$. A proper $k$-vertex-coloring of a graph $G$ is a function from $V(G)$ to the set of colors $\llbracket k \rrbracket=\{1, \ldots, k\}$, such that no two adjacent vertices receive the same color. The chromatic number $\chi(G)$ of a graph $G$ is the smallest $k$ such that $G$ admits a proper $k$-vertex-coloring.

A homomorphism of $G$ to $H$ is a function $\varphi$ from $V(G)$ to $V(H)$ such that for all $x, y \in V(G), x y \in E(G)$ implies $\varphi(x) \varphi(y) \in E(H)$. When there is a homomorphism of $G$ to $H$, we note $G \rightarrow H$. Note that the chromatic number of $G, \chi(G)$, can also be defined as the smallest order of a graph $H$ such that $G \rightarrow H$. An isomorphism of $G$ to $H$ is a bijection $\varphi$ from $V(G)$ to $V(H)$ such that for all $x, y \in V(G), x y \in E(G)$ if and only if $\varphi(x) \varphi(y) \in E(H)$. In this case, we note $G=H$.

A walk in a graph $G$ is a sequence $s_{0}, \ldots, s_{n}$ of vertices of $G$ such that $s_{i} s_{i+1} \in E(G)$. Its starting vertex is $s_{0}$ and its end vertex is $s_{n}$. A closed walk is a walk where $s_{0}=s_{n}$. If all elements of a walk are pairwise distinct, then the walk is a path. A closed walk where all elements are pairwise distinct, except $s_{0}$ and $s_{n}$, is a cycle. The length (number of edges, counted with multiplicity) of a walk $W=s_{0}, \ldots, s_{n}$ is $n$, and its order (number of vertices, counted with multiplicity) is $n$ if $W$ is a closed walk, or $n+1$ otherwise.

A graph is connected if for all pairs of vertices $u, v \in V(G)$, there is a path between $u$ and $v$. If $X \subseteq V(G)$, then the graph $G[X]$ is the subgraph of $G$ induced by $X$. We say that $G[X]$ is an induced subgraph of $G$. The complete graph $K_{p}$ is the graph of order $p$ such that for all pair of distinct vertices of $G, u$ and $v, u v$ is an edge of $K_{p}$.

A signed graph $(G, \sigma)$ is a graph $G$ along with a function $\sigma: E(G) \rightarrow\{+1,-1\}$ called the signature of $(G, \sigma)$, where $\sigma(e)$ is the sign of the edge $e \in E(G)$. The edges in $\sigma^{-1}(+1)$ are the positive edges and the edges in $\sigma^{-1}(-1)$ are the negative edges of $(G, \sigma)$. We often write a signed graph $(G, \sigma)$ as $(G, \Sigma)$ where $\Sigma$ is the set of negative edges, that is $\Sigma=\sigma^{-1}(-1)$. These two ways to represent a signed graph are equivalent 
and will be used interchangeably. We note $K_{p}^{+}$(resp. $K_{p}^{-}$) for the complete signed graph $\left(K_{p}, \varnothing\right)$ (resp. $\left.\left(K_{p}, E\left(K_{p}\right)\right)\right)$ of order $p$ with only positive (resp. negative) edges.

Let $(G, \sigma)$ be a signed graph and $v$ be a vertex of $G$. To switch $v$ is to create the signed graph $\left(G, \sigma^{\prime}\right)$ where $\sigma^{\prime}(e)=-\sigma(e)$ when $e$ is incident to $v$ and $\sigma^{\prime}(e)=\sigma(e)$ otherwise. To switch a set $X$ of vertices of $(G, \sigma)$ is to create the signed graph $\left(G, \sigma^{\prime}\right)$ where $\sigma^{\prime}$ is obtained by switching every vertex of $X$, in any order. This led Zaslavsky in [18] to define the notion of equivalent signed graphs. Two signed graphs $\left(G, \sigma_{1}\right)$ and $\left(G, \sigma_{2}\right)$ on the same underlying graph are equivalent if there exists a set $X \subseteq V(G)$ such that $\left(G, \sigma_{2}\right)$ is obtained from $\left(G, \sigma_{1}\right)$ by switching $X$. In this case we note $\left(G, \sigma_{1}\right) \equiv\left(G, \sigma_{2}\right)$. We also say that the two signatures $\sigma_{1}$ and $\sigma_{2}$ (resp. $\Sigma_{1}$ and $\Sigma_{2}$ ) are equivalent and we note $\sigma_{1} \equiv \sigma_{2}\left(\right.$ resp. $\left.\Sigma_{1} \equiv \Sigma_{2}\right)$.

Suppose that $(G, \sigma)$ is a signed graph and $W$ is a walk $s_{0}, \ldots, s_{n}$ in $G$. We say that $W$ is a balanced walk if $\sigma(W)=\sigma\left(s_{0} s_{1}\right) \sigma\left(s_{1} s_{2}\right) \ldots \sigma\left(s_{i} s_{i+1}\right) \ldots \sigma\left(s_{n-1} s_{n}\right)=1$ and an unbalanced walk otherwise. Similarly, this notion can be extended to closed walks, paths and cycles. We note an unbalanced path (resp. balanced path) of order $k$ by $U P_{k}$ (resp. $B P_{k}$ ) and an unbalanced cycle (resp. balanced cycle) of order $k$ by $U C_{k}$ (resp. $B C_{k}$ ). A signed graph where all closed walks are balanced is said to be balanced while a signed graph where all closed walks are unbalanced is said to be antibalanced. Generally, for the same ordinary graph $G$, there are several signatures $\sigma$ for which $(G, \sigma)$ is balanced. They are precisely the signatures $\sigma_{X}$ which can be obtained from $(G, \varnothing)$ by switching $X$, where $X \subseteq V(G)$. In particular it is the case for all signatures of a forest. These notions of balanced and antibalanced graphs where introduced by Harary in [8].

One can check that the switch operation does not modify the set of balanced closed walks as switching at a vertex of a closed walk does not change the sign of this walk. Hence, signed graphs $\left(G, \sigma_{1}\right)$ and $\left(G, \sigma_{2}\right)$ on the same underlying graph are equivalent if and only if they have the same set of balanced closed walks [18]. Note that this is equivalent to having the same set of balanced cycles, or the same set of unbalanced closed walks (resp. cycles). This means that we can work with the balance of closed walks or with switches depending on which notion is the easiest to use when treating equivalence of signed graphs.

A homomorphism of a signed graph $(G, \sigma)$ to a signed graph $(H, \pi)$ is a homomorphism $\varphi$ of $G$ to $H$ which maps balanced (resp. unbalanced) closed walks of $(G, \sigma)$ to balanced (resp. unbalanced) closed walks of $(H, \pi)$. Alternatively, a homomorphism of $(G, \sigma)$ to $(H, \pi)$ is a homomorphism of $G$ to $H$ such that there exists a signature $\sigma^{\prime}$ of $G$ with $\sigma^{\prime} \equiv \sigma$, such that if $e$ is an edge of $G$, then $\pi(\varphi(e))=\sigma^{\prime}(e)$. When there is a homomorphism of $(G, \sigma)$ to $(H, \pi)$, we note $(G, \sigma) \longrightarrow_{s}(H, \pi)$ and say that $(G, \sigma)$ maps to $(H, \pi)$. Here $(H, \pi)$ is the target graph of the homomorphism. When constructing a homomorphism, we can always fix a given signature of the target graph [13].

The chromatic number $\chi_{s}(G, \sigma)$ of a signed graph $(G, \sigma)$ is the smallest $k$ for which $(G, \sigma)$ admits a homomorphism to a signed graph $(H, \pi)$ of order $k$. Alternatively, a signed graph $(G, \sigma)$ admits a $k$-(vertex)-coloring if there exists $\sigma^{\prime} \equiv \sigma$ such that $\left(G, \sigma^{\prime}\right)$ admits a proper vertex coloring $\theta: V(G) \rightarrow \llbracket k \rrbracket$ verifying that for every $i, j \in \llbracket k \rrbracket$, all edges $u v$ with $\theta(u)=i$ and $\theta(v)=j$ have the same sign in $\left(G, \sigma^{\prime}\right)$. Here $\chi_{s}(G, \sigma)$ is the smallest $k$ such that $(G, \sigma)$ admits a $k$-vertex-coloring. The two definitions are equivalent, as with any coloring of a signed graph, we can associate a homomorphism of 
signed graphs which identifies the vertices with the same color. The homomorphism is well defined as long as the target graph is simple, which is the case here by definition of a $k$-vertex-coloring.

The Cartesian product of two ordinary graphs $G$ and $H$ is the graph $G \square H$ whose vertex set is $V(G) \times V(H)$ and where $(x, y)$ and $\left(x^{\prime}, y^{\prime}\right)$ are adjacent if and only if $x=x^{\prime}$ and $y$ is adjacent to $y^{\prime}$ in $H$, or $y=y^{\prime}$ and $x$ is adjacent to $x^{\prime}$ in $G$.

A graph $G$ is prime if there are no graphs $A$ and $B$ on at least two vertices for which $G=A \square B$. A decomposition $D$ of a graph $G$ is a multiset $\left\{G_{1}, \ldots, G_{k}\right\}, k \geq 1$, such that the $G_{i}$ 's are graphs containing at least one edge and $G=G_{1} \square \ldots \square G_{k}$. A decomposition is prime if all the $G_{i}$ 's are prime. The $G_{i}$ 's are called factors of $G$. A decomposition $D^{\prime}$ is finer than a decomposition $D=\left\{G_{1}, \ldots, G_{k}\right\}$, if for all $i \in \llbracket k \rrbracket$, there is a decomposition $D_{i}^{\prime}=\left\{G_{i, 1}^{\prime}, \ldots, G_{i, p_{i}}^{\prime}\right\}$ of $G_{i}$ such that $D^{\prime}=\left\{G_{1,1}^{\prime}, \ldots, G_{1, p_{1}}^{\prime}, G_{2,1}^{\prime}, \ldots, G_{k, p_{k}}^{\prime}\right\}$. Note that by definition, every decomposition is finer than itself.

Suppose that $G$ is a graph and $D=\left\{G_{1}, \ldots, G_{k}\right\}$ is a decomposition of $G$ such that $G=G_{1} \square \ldots \square G_{k}$. A coordinate system for $G$ under the decomposition $D$ is a bijection $\theta: V(G) \rightarrow \prod_{i=1}^{k} V\left(G_{i}\right)$ verifying that for each vertex $v$ of $G$, the set of vertices which differ from $v$ by the $i$ th coordinate induces a graph, noted $G_{i}^{v}$ and called a $G_{i}$-layer, which is isomorphic to $G_{i}$ by the projection on the $i$ th coordinate. An edge $u v$ of $G$ is a copy of an edge $a b$ of $G_{i}$ if $\theta(u)$ and $\theta(v)$ differ only in their $i$ th coordinate with $u_{i}=a$ and $v_{i}=b$. For a vertex $u$ of $G$ and a $G_{i}$-layer $G_{i}^{v}$, the projection of $u$ on the $G_{i}$-layer $G_{i}^{v}$ is the vertex $w$ of $V\left(G_{i}^{v}\right)$ which is the closest to $u$.

Suppose $D=\left\{G_{1}, \ldots, G_{k}\right\}$ is a decomposition of an ordinary graph $G$. We say that two $G_{i}$-layers $X_{1}$ and $X_{2}$ are adjacent by $G_{j}$ if and only if there exists an edge $a b$ of a $G_{j}$-layer such that $a \in X_{1}$ and $b \in X_{2}$. In other words, the subgraph induced by the vertices of $X_{1}$ and $X_{2}$ is isomorphic to $G_{i} \square K_{2}$ where $K_{2}$ corresponds to the edge $a b$.

Let $A$ and $B$ be two ordinary graphs. The greatest common divisor of $A$ and $B$ is the graph $X$ such that, for every three graphs $W, Y$, and $Z$ with $A=W \square Y$ and $B=W \square Z, X$ is a factor of $W$.

\section{Preliminary results}

The goal of this section is to present useful results on signed graphs and on the Cartesian product of ordinary graphs.

In [18], Zaslavsky gave a way to determine if two signed graphs are equivalent in linear time. In particular, all signed forests with the same underlying graph are equivalent. This theorem comes from the following observation.

Observation 1 (Zaslavsky [18]). If $C$ is a cycle of a graph $G$, then switching any number of vertices of $G$ does not change the parity of the number of negative edges of $C$.

This implies that we can separate the set of all cycles into four families $B C_{\text {even }}$, $B C_{o d d}, U C_{\text {even }}$ and $U C_{\text {odd }}$, depending on the parity of the number of negative edges (even for $B C_{\text {even }}$ and $B C_{\text {odd }}$ and odd for $U C_{\text {even }}$ and $U C_{o d d}$ ) and the parity of the length of the cycle (even for $B C_{\text {even }}$ and $U C_{\text {even }}$ and odd for $B C_{\text {odd }}$ and $U C_{\text {odd }}$ ).

Theorem 2. Let $(C, \sigma)$ be a signed cycle. We then have: 
1. $\chi_{s}(C, \sigma)=2$ if $(C, \sigma) \in B C_{\text {even }}$,

2. $\chi_{s}(C, \sigma)=3$ if $(C, \sigma) \in B C_{\text {odd }} \cup U C_{\text {odd }}$,

3. $\chi_{s}(C, \sigma)=4$ if $(C, \sigma) \in U C_{\text {even }}$.

Proof. By [3], we already have the upper bounds. A homomorphism of signed graphs is also a homomorphism of graphs thus $\chi(C) \leq \chi_{s}(C, \sigma)$. This proves the lowers bounds for the first two cases. Let $(C, \sigma) \equiv U C_{2 q}$ and suppose $\chi_{s}(C, \sigma) \leq 3$. Then $(C, \sigma) \longrightarrow_{s}$ $\left(K_{3}, \pi\right)$. In each case, $\left(K_{3}, \pi\right)$ can be switched either to be all positive or to be all negative. This means that $(C, \sigma)$ can be switched either to be all positive or to be all negative, which is not the case as $U C_{2 q}$ has an odd number of negatives edges and an odd number of positive edges, a contradiction. We get the desired lower bounds in each case.

One of the first results on the chromatic number of Cartesian products of ordinary graphs is due to Sabidussi:

Theorem 3 (Sabidussi [14]). For every two graphs $G$ and $H, \chi(G \square H)=$ $\max (\chi(G), \chi(H))$.

Following this paper, Sabidussi proved one of the most important results on the Cartesian product: the unicity of the prime decomposition of connected graphs. This result was independently proved by Vizing.

Theorem 4 (Sabidussi [15] and Vizing [16]). Every connected ordinary graph G admits a unique prime decomposition up to the order and isomorphisms of the factors.

Using some arguments of [6] and the previous theorem, Imrich and Klavžar proved the following theorem.

Theorem 5 (Imrich and Klavžar $[9,10]$ ). If $A, B$ and $C$ are three ordinary graphs such that $A \square B=A \square C$, then $B=C$.

The unicity of the prime decomposition raises the question of the complexity of finding such a decomposition. The complexity of decomposition algorithms has been extensively studied. The first algorithm, by Feigenbaum et al. [5], had a complexity of $O\left(n^{4.5}\right)$ where $n$ is the order of the graph (its size is denoted by $m$ ). In [17], Winkler proposed a different algorithm improving the complexity to $O\left(n^{4}\right)$. Then Feder [4] gave an algorithm in $O(m n)$ time and $O(m)$ space. The same year, Aurenhammer et al. [1] gave an algorithm in $O(m \log n)$ time and $O(m)$ space. The latest result is an optimal algorithm.

Theorem 6 (Imrich and Peterin [11]). The prime factorization of connected ordinary graphs can be found in $O(m)$ time and space. Additionally a coordinate system can be computed in $O(m)$ time and space.

\section{Cartesian products of signed graphs}

\subsection{Definition}

We recall the definition of the Cartesian product of signed graphs due to Germina, Hameed K. and Zaslavsky: 
Definition $7([7])$. Let $(G, \sigma)$ and $(H, \pi)$ be two signed graphs. The Cartesian product of $(G, \sigma)$ and $(H, \pi)$, denoted by $(G, \sigma) \square(H, \pi)$, is the signed graph defined as follows:

- $V((G, \sigma) \square(H, \pi))=V(G) \times V(H)$,

- the positive (resp. negative) edges are the pairs $\left\{\left(u_{1}, v_{1}\right),\left(u_{2}, v_{2}\right)\right\}$ such that:

- $u_{1}=u_{2}$ and $v_{1} v_{2}$ is a positive (resp. negative) edge of $(H, \pi)$, or

$-v_{1}=v_{2}$ and $u_{1} u_{2}$ is a positive (resp. negative) edge of $(G, \sigma)$.

Note that the underlying graph of $(G, \sigma) \square(H, \pi)$ is the ordinary graph $G \square H$. From this definition, we can derive that the Cartesian product is associative and commutative.

The following result shows that Cartesian products are compatible with homomorphisms of signed graphs and in particular with the switching operation.

Theorem 8. If $(G, \sigma),\left(G^{\prime}, \sigma^{\prime}\right),(H, \pi),\left(H^{\prime}, \pi^{\prime}\right)$ are four signed graphs such that $(G, \sigma) \longrightarrow_{s}\left(G^{\prime}, \sigma^{\prime}\right)$ and $(H, \pi) \longrightarrow_{s}\left(H^{\prime}, \pi^{\prime}\right)$, then:

$$
(G, \sigma) \square(H, \pi) \longrightarrow_{s}\left(G^{\prime}, \sigma^{\prime}\right) \square\left(H^{\prime}, \pi^{\prime}\right) .
$$

Proof. By commutativity of the Cartesian product and composition of homomorphisms, it suffices to show that $(G, \sigma) \square(H, \pi) \longrightarrow_{s}\left(G^{\prime}, \sigma^{\prime}\right) \square(H, \pi)$. Since $(G, \sigma) \longrightarrow_{s}$ $\left(G^{\prime}, \sigma^{\prime}\right)$, there exists a set $S$ of vertices and a homomorphism $\varphi$ of $G$ to $G^{\prime}$ such that if $\left(G, \sigma_{S}\right)$ is the signed graph obtained from $(G, \sigma)$ by switching the vertices of $S$, then $\sigma^{\prime}(\varphi(e))=\sigma_{S}(e)$ for every edge $e$ of $G$. We note $P=(G, \sigma) \square(H, \pi)$ and $X=\{(g, h) \in V(G \square H) \mid g \in S\}$. Let $P^{\prime}$ be the signed graph obtained from $P$ by switching the vertices in $X$.

If $(g, h)\left(g, h^{\prime}\right)$ is an edge of $P$, then in $P^{\prime}$ this edge was either switched twice if $g \in S$ or not switched if $g \notin S$. In both cases its sign did not change. If $(g, h)\left(g^{\prime}, h\right)$ is an edge of $P$, then in $P^{\prime}$ this edge was switched twice if $g, g^{\prime} \in S$, switched once if $g \in S, g^{\prime} \notin S$ or $g \notin S, g^{\prime} \in S$, and not switched if $g, g^{\prime} \notin S$. In each case its new sign is $\sigma_{S}\left(g g^{\prime}\right)$. Thus $P^{\prime}=\left(G, \sigma_{S}\right) \square(H, \pi)$. Now define $\varphi_{P}(g, h)=(\varphi(g), h)$. It is a homomorphism of $G \square H$ to $G^{\prime} \square H$ by definition. By construction, the target graph of $\varphi_{P}$ is $\left(G^{\prime}, \sigma^{\prime}\right) \square(H, \pi)$ as the edges of $H$ do not change and the target graph of $\varphi$ is $\left(G^{\prime}, \sigma^{\prime}\right)$.

As mentioned before, we can derive the following corollary from Theorem 8 .

Corollary 9. If $(G, \sigma),\left(G, \sigma^{\prime}\right),(H, \pi),\left(H, \pi^{\prime}\right)$ are four signed graphs such that $\sigma \equiv \sigma^{\prime}$ and $\pi \equiv \pi^{\prime}$, then:

$$
(G, \sigma) \square(H, \pi) \equiv\left(G, \sigma^{\prime}\right) \square\left(H, \pi^{\prime}\right) .
$$

From Theorem 8, and the fact that $(F, \sigma) \longrightarrow_{s} K_{2}^{+}$for every signed forest $(F, \sigma)$, we also get the following corollary:

Corollary 10. If $(G, \sigma)$ is a signed graph and $(F, \pi)$ is a signed forest with at least one edge, then:

$$
\chi_{s}((G, \sigma) \square(F, \pi))=\chi_{s}\left((G, \sigma) \square K_{2}^{+}\right) .
$$

In particular, for $n, m \geq 2, \chi_{s}\left(\left(P_{n}, \sigma_{1}\right) \square\left(P_{m}, \sigma_{2}\right)\right)=2$. 


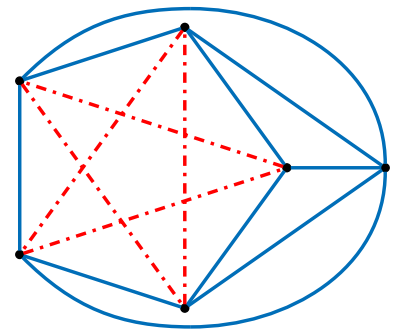

(a) The graph $\mathrm{SPal}_{5}^{*}$.

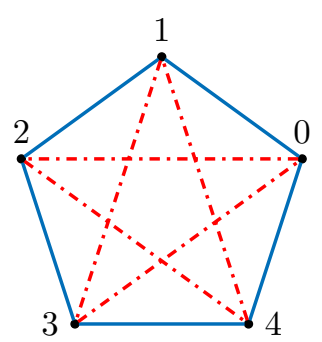

(b) The graph $\mathrm{SPal}_{5}$.

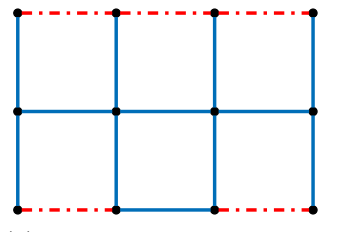

(c) A grid $G$ with $\chi_{s}(G)=5$

Figure 1: The signed graphs used in the proof of Theorem 11.

\subsection{Signed grids}

Note that there is a difference between considering the chromatic number of the Cartesian product of two signed graphs and the chromatic number of a signed graph whose underlying graph is a Cartesian product. For example, $C_{4}=K_{2} \square K_{2}$ but $4=\chi_{s}\left(U C_{4}\right) \neq$ $\chi_{s}\left(B C_{4}\right)=2$. Another example comes from grid graphs: $\chi_{s}\left(\left(P_{n}, \sigma_{1}\right) \square\left(P_{m}, \sigma_{2}\right)\right)=2$, for any $n, m \in \mathbb{N}$, but the following theorem shows that not all signed grids have chromatic number 2 .

Theorem 11. If $n$ and $m$ are two integers with $1 \leq n \leq m$ and $(G, \sigma)$ is a signed grid with $G=P_{n} \square P_{m}$, then $\chi_{s}(G) \leq 6$. If $n \leq 4$, then $\chi_{s}(G) \leq 5$. Moreover there exist signed grids with chromatic number 5 .

On our figures, we use dashed red edges to represent negative edges and solid blue edges for positive edges.

Proof. We will prove a more precise statement: every signed grid $(G, \sigma)$ verifies $(G, \sigma) \longrightarrow_{s} S P a l_{5}^{*}$ where $S P_{a l}^{*}$ is the graph of Figure 1a. This graph has the following (easy to check) property:

$(\mathcal{P})$ for every three vertices $x, y, z$ of $S P a l_{5}^{*}$, and every $\operatorname{sign} \epsilon \in\{+1,-1\}$, if $x \neq z$ or $\epsilon=+1$ then there exists $u$ and $v$ in $S P a l_{5}^{*}, u \neq v$, such that the cycles $x y z u$ and $x y z v$ have $\operatorname{sign} \epsilon$.

To map $(G, \sigma)$ to $S P a l_{5}^{*}$, we will construct the homomorphism $\varphi$ by induction. The vertex of $G$ in line $i \in\{1, \ldots, n\}$ and column $j \in\{1, \ldots, m\}$ will be called $x_{i, j}$. Let $H_{i, j}$ be the subgraph of $G$ induced by the vertices $x_{k, \ell}$ where $k<i$, or $k=i$ and $\ell \leq j$. We prove that for all $i, j, 0 \leq i \leq n$ and $0 \leq j \leq m, H_{i, j} \longrightarrow_{s} S p a l_{5}^{*}$. It is easy to see that $H_{0, m} \longrightarrow_{s} \mathrm{SPal}_{5}^{*}$.

If $H_{i, m} \longrightarrow_{s} S p a l_{5}^{*}$ and $i<n$, then $x_{i+1,0}$ has only one neighbor in $H_{i+1,0}$ and we can extend the previous homomorphism to $H_{i+1,0}$.

Suppose that $\varphi$ is a homomorphism of $H_{i, j}$ to $S p a l_{5}^{*}$ and $j<m$. Let $C=$ $x_{i, j+1} x_{i, j} x_{i-1, j} x_{i-1, j+1}$. If $C=B C_{4}$ or if $C=U C_{4}$ and $\varphi\left(x_{i, j}\right) \neq \varphi\left(x_{i-1, j+1}\right)$, then we have two choices for $x_{i, j+1}$ by $\mathcal{P}$ (we might need to switch $x_{i, j}$ ) and we can extend the homomorphism to $H_{i, j+1}$. If $C=U C_{4}$ and $\varphi\left(x_{i, j}\right)=\varphi\left(x_{i-1, j+1}\right)$, then these two vertices must be different. There were two possibilities for the choice of $\varphi\left(x_{i, j-1}\right)$ in the 
previous step by $\mathcal{P}$ thus if we take the other one, we are back to the previous case where $\varphi\left(x_{i, j-1}\right) \neq \varphi\left(x_{i-1, j}\right)$. Thus we can extend $\varphi$ to $H_{i, j+1}$.

Hence, $H_{n, m}=(G, \sigma) \longrightarrow_{s} S P a l_{5}^{*}$ which gives $\chi_{s}(G, \sigma) \leq 6$.

Suppose now that $n \leq 4$. We construct a homomorphism $\varphi:(G, \sigma) \longrightarrow_{s} \mathrm{SPal}_{5}$, column by column, where $S \mathrm{Pal}_{5}$ is the graph of Figure 1b. The first column is a path and thus, we can map it arbitrarily to $S P a l_{5}$. For a column with vertices $x_{1, j}, x_{2, j}, x_{3, j}, x_{4, j}$, there are at least three possibilities to map $x_{1, j}$ (the three colors different from $\varphi\left(x_{1, j-1}\right)$ and $\left.\varphi\left(x_{2, j-1}\right)\right)$. Up to symmetry, we can suppose that $x_{1, j-1}$ has color 0 and $x_{1, j-1}$ has color 1 . These three possibilities $(2,3,4)$ give at least three possibilities for $x_{2, j}$ where we need to remove one of them to account for the possibility of a $U C_{4}$ forcing $x_{2, j}$ and $x_{3, j-1}$ to have different images. Indeed, let $C=x_{1, j} x_{2, j} x_{2, j-1} x_{1, j-1}$, if $C=B C_{4}$ then $x_{2, j}$ can have colors 1,2 or 3 . If $C=U C_{4}$, then $x_{2, j}$ can have colors 2,3 or 4 .

Again for $x_{3, j}$ there are at least two possibilities by the same kind of arguments. We need to remove one of them to account for the possibility of a $U C_{4}$ forcing $x_{3, j}$ and $x_{4, j-1}$ to have different colors. Finally there is at least one possibility for $x_{4, j}$. Thus we can extend our homomorphism to this column. This implies that $G \longrightarrow_{s} S P a l_{5}$.

It is tedious but not difficult to check that the signed grid of Figure 1c cannot be mapped to a signed graph of order 4 , thus its chromatic number if at least 5 . In fact it is exactly 5 . This concludes the proof.

The arguments for the existence of a homomorphism to $\mathrm{SPal}_{5}$ cannot be extended to bigger grids as we could end up in the case where $x_{4, j}$ has no possible image. We do not know if the upper bound for grids is 5 or 6 .

Question 12. What is the maximal value of $\chi_{s}(G, \sigma)$ when $(G, \sigma)$ is a signed grid? Is it 5 or 6 ?

\subsection{Prime factor decomposition}

Our goal now is to prove that each connected signed graph has a unique prime $s$ decomposition. Let us start with some definitions.

Definition 13. A signed graph $(G, \sigma)$ is said to be s-prime if and only if there do not exist two signed graphs $\left(A, \pi_{A}\right)$ and $\left(B, \pi_{B}\right)$ such that $(G, \sigma) \equiv\left(A, \pi_{A}\right) \square\left(B, \pi_{B}\right)$. An $s$-decomposition of a signed connected graph $(G, \sigma)$ is a multiset of signed graphs $D=\left\{\left(G_{1}, \pi_{1}\right), \ldots,\left(G_{k}, \pi_{k}\right)\right\}$ such that:

1. the $\left(G_{i}, \pi_{i}\right)$ 's are signed graphs containing at least one edge and

2. $(G, \pi) \equiv\left(G_{1}, \pi_{1}\right) \square \cdots \square\left(G_{k}, \pi_{k}\right)$.

An $s$-decomposition $D$ is prime if all the $\left(G_{i}, \pi_{i}\right)$ 's are $s$-prime. The $\left(G_{i}, \pi_{i}\right)$ 's are called factors of $D$.

Note that if $G=A \square B$, then it is not always true that $(G, \sigma)$ is the Cartesian product of two signed graphs. For example, $U C_{4}$ is $s$-prime but $C_{4}$ is not a prime graph as $C_{4}=K_{2} \square K_{2}$. The following lemma tells us in which cases $(G, \sigma) \equiv\left(A, \pi_{A}\right) \square\left(B, \pi_{B}\right)$, and will be a useful tool for decomposing signed graphs.

Lemma 14. If $(G, \sigma),\left(A, \pi_{A}\right)$ and $\left(B, \pi_{B}\right)$ are three connected signed graphs with $G=$ $A \square B$, then $(G, \sigma) \equiv\left(A, \pi_{A}\right) \square\left(B, \pi_{B}\right)$ if and only if: 
1. all A-layers are equivalent to $\left(A, \pi_{A}\right)$,

2. at least one $B$-layer is equivalent to $\left(B, \pi_{B}\right)$, and

3. for each edge $e$ of $A$ and each pair of distinct copies $e_{1}, e_{2}$ of $e$, if $e_{1}$ and $e_{2}$ belong to the same 4-cycle, then this cycle is a $B C_{4}$.

Note that, in the previous lemma, all $B$-layers are equivalent to $\left(B, \pi_{B}\right)$ but we only need to verify that for one of them to conclude.

Proof. $(\Rightarrow)$ This follows from the definition of the Cartesian product.

$(\Leftarrow)$ We will do the following independent switches: switch all $A$-layers to have the same signature $\pi_{A}$.

Now we claim that all $B$-layers have the same signature $\pi_{B}^{\prime}$ equivalent to $\pi_{B}$. Indeed take one edge $x y$ of $B$ and two copies of this edge $x_{1} y_{1}$ and $x_{2} y_{2}$ in $G$. Take a shortest path $P$ from $x_{1}$ to $x_{2}$ in the $A^{x_{1}}$-layer. Now if $u_{1}, u_{2}$ are two consecutive vertices along $P$ and $v_{1}$ and $v_{2}$ are their projections on $A^{y_{1}}$, then $u_{1} u_{2} v_{2} v_{1}$ is a $B C_{4}$ by the third hypothesis as $u_{1} v_{1}$ and $u_{2} v_{2}$ are copies of the edge $x y$.

As $u_{1} u_{2}$ and $v_{1} v_{2}$ have the same sign by the previous switches, it must be that $u_{1} v_{1}$ and $u_{2} v_{2}$ have the same sign. Thus all copies of an edge of $B$ have the same sign.

Hence, $(G, \sigma) \equiv\left(A, \pi_{A}\right) \square\left(B, \pi_{B}^{\prime}\right) \equiv\left(A, \pi_{A}\right) \square\left(B, \pi_{B}\right)$ by Theorem 8 .

One of our main results is the following Prime Decomposition Theorem.

Theorem 15 (Prime Decomposition Theorem). If $(G, \sigma)$ is a connected signed graph and $D$ is the prime decomposition of $G$, then $(G, \sigma)$ admits a unique (up to isomorphism and order of the factors) prime s-decomposition $D_{s}$. Moreover, if we see $D_{s}$ as a decomposition of $G$, then $D$ is finer than $D_{s}$.

For proving this theorem, we need the following lemma.

Lemma 16. If $(G, \sigma)$ is a connected signed graph that admits two prime s-decompositions $D_{1}$ and $D_{2}$, then there are two signed graphs $\left(X, \pi_{X}\right)$ and $\left(Y, \pi_{Y}\right)$ such that $(G, \sigma) \equiv$ $\left(X, \pi_{X}\right) \square\left(Y, \pi_{Y}\right)$ with $D_{1}=\left\{\left(X, \pi_{X}\right)\right\} \cup D_{1}^{\prime}$ and $D_{2}=\left\{\left(X, \pi_{X}\right)\right\} \cup D_{2}^{\prime}$, where $D_{1}^{\prime}$ and $D_{2}^{\prime}$ are two s-decompositions of $\left(Y, \pi_{Y}\right)$.

Proof. Suppose there exists a signed graph $(G, \sigma)$ that admits two $s$-decompositions $D_{1}$ and $D_{2}$. Fix an edge $e$ of $(G, \sigma)$ which belongs to some $Z$-layer $Z^{e}$ of the prime decomposition of $G$. The edge $e$ belongs to some $\left(A, \pi_{A}\right)$-layer in $D_{1}$ and to some $\left(B, \pi_{B}\right)$-layer in $D_{2}$. The graph $Z$ is a factor of $A$ and $B$ by unicity of the prime factor decomposition of $G$. Let $X$ be the greatest common divisor of $A$ and $B$. Since $e \in E\left(Z^{e}\right), e$ is in some $X$-layer $X^{e}$. Now $G=X \square Y$ for some graph $Y$. Let us show that $(G, \sigma) \equiv\left(X, \pi_{X}\right) \square\left(Y, \pi_{Y}\right)$ for some signatures $\pi_{X}$ and $\pi_{Y}$ of $X$ and $Y$, respectively. We can suppose that $Y \neq K_{1}$ and $A \neq B$, as otherwise the result is immediate.

First we want to show that all $X$-layers have equivalent signatures. Take two adjacent $X$-layers. If they are in different $A$-layers, then they are equivalent since they represent the same part of $\left(A, \pi_{A}\right)$. If they are in the same $A$-layer, then they are in different $B$ layers since $X$ is the greatest common divisor of $A$ and $B$. The same argument works in this case. Thus two adjacent $X$-layers are isomorphic to the same signed $\operatorname{graph}\left(X, \pi_{X}\right)$, and since there is only one connected component in $Y$, all $X$-layers have equivalent signatures. 
Let $\pi_{Y}$ be the signature of one $Y$-layer. Fix $e^{\prime}$ an edge of $X$, and $X_{1}, X_{2}$ two $X$ layers. Now consider the 4-cycle (if it exists) containing the copies of this edge in each of the two layers. If $X_{1}$ and $X_{2}$ are in different $A$-layers, then this cycle is a $B C_{4}$ by Lemma 14, otherwise this cycle is a $B C_{4}$ as $X_{1}$ and $X_{2}$ are in different $B$-layers by the same argument.

By Lemma 14 , we can conclude that $(G, \sigma) \equiv\left(X, \pi_{X}\right) \square\left(Y, \pi_{Y}\right)$.

Now suppose that $A=X \square W$. Using Lemma 14 , we can show that $\left(A, \pi_{X}\right) \equiv$ $\left(X, \pi_{X}\right) \square\left(W, \pi_{W}\right)$. Indeed all $X$-layers have equivalent signatures since $(G, \sigma) \equiv$ $\left(X, \pi_{X}\right) \square\left(Y, \pi_{Y}\right)$ and all 4-cycles between two copies of an edge of $X$ are $B C_{4}$ by the same argument. As $\left(A, \pi_{A}\right)$ is $s$-prime, this implies $\left(X, \pi_{X}\right) \equiv\left(A, \pi_{A}\right)$. Thus $\left(X, \pi_{X}\right) \equiv\left(A, \pi_{A}\right) \equiv\left(B, \pi_{B}\right)$ and this proves the lemma.

Proof of Theorem 15. Any signed graph $(G, \sigma)$ has a prime $s$-decomposition by taking an $s$-decomposition that cannot be refined. Every prime $s$-decomposition of $(G, \sigma)$ can be considered as a decomposition of $G$, and the prime decomposition of $G$ is finer than every such decomposition. We still have to show that the prime $s$-decomposition of $(G, \sigma)$ is unique. Suppose, to the contrary, that $(G, \sigma)$ is a minimal counterexample to the unicity. Thus $(G, \sigma)$ has two prime $s$-decompositions $D_{1}$ and $D_{2}$ and, by Lemma 16 , $(G, \sigma) \equiv\left(X, \pi_{X}\right) \square\left(Y, \pi_{Y}\right)$ with $D_{1}=\left\{\left(X, \pi_{X}\right)\right\} \cup D_{1}^{\prime}$ and $D_{2}=\left\{\left(X, \pi_{X}\right)\right\} \cup D_{2}^{\prime}$, where $D_{1}^{\prime}$ and $D_{2}^{\prime}$ are two s-decompositions of $\left(Y, \pi_{Y}\right)$. By minimality of $(G, \sigma),\left(Y, \pi_{Y}\right)$ has a unique prime s-decomposition, hence $D_{1}^{\prime}=D_{2}^{\prime}$. Thus $D_{1}=D_{2}$, a contradiction.

Note that Theorem 15 implies the following result.

Theorem 17. If $\left(A, \pi_{A}\right),\left(B, \pi_{B}\right)$ and $\left(C, \pi_{C}\right)$ are three signed graphs verifying $\left(A, \pi_{A}\right) \square\left(B, \pi_{B}\right) \equiv\left(A, \pi_{A}\right) \square\left(C, \pi_{C}\right)$, then $\left(B, \pi_{B}\right) \equiv\left(C, \pi_{C}\right)$.

The proof of this result is exactly the same as the proof for ordinary graphs presented in [10]. Indeed, we have all the necessary tools used in the proof. The first one is Theorem 15, the other one is the semi-ring structure of signed graphs (quotiented by the equivalence relation) with the disjoint union and the Cartesian product which follows from the definition. See [10] for more details on the proof.

\subsection{Recognising Cartesian products of signed graphs}

In the last part of this section, we propose an algorithm to decompose connected signed graphs. Decomposing a graph can be interpreted in multiple ways: finding a decomposition, identifying which edge of $G$ belongs to which factor, or even better getting a coordinate system that is compatible with the decomposition. In [11], Imrich and Peterin gave an $O(m)$ time and space ( $m$ is the number of edges of $G$ ) algorithm for these three questions for ordinary graphs. More recently, in [12], they gave another algorithm in $O(m)$ time and space to decompose directed graphs.

Our goal is to give a similar algorithm for signed graphs based on their algorithm for directed graphs.

Theorem 18. Let $(G, \sigma)$ be a connected signed graph of order $n$ and size $m$. We can find in time $O(m)$ and space $O(m)$ the prime s-decomposition of $(G, \sigma)$ and a coordinate system for this decomposition. 


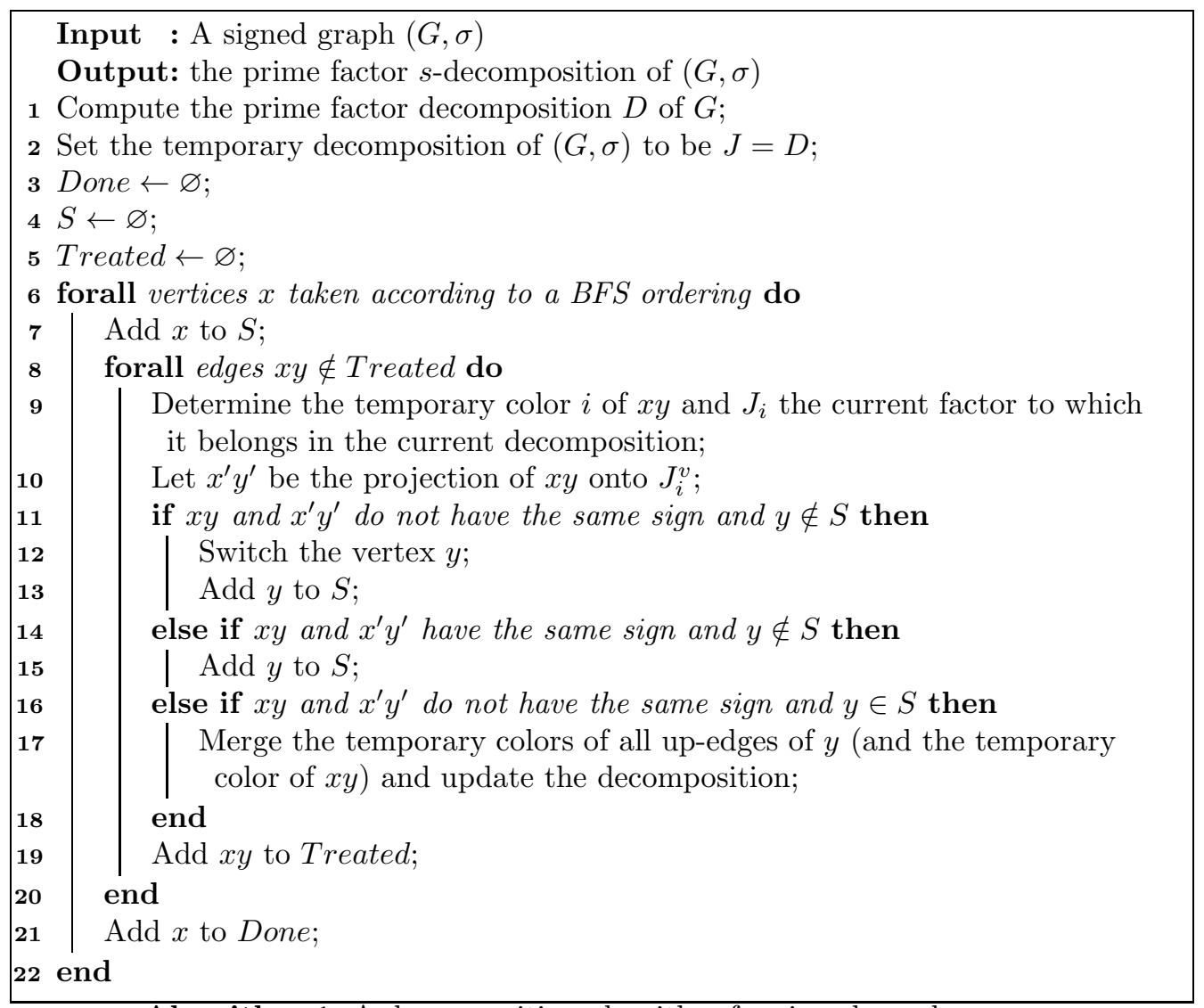

Algorithm 1: A decomposition algorithm for signed graphs.

We take a coordinate system for a graph $G$ corresponding to its prime decomposition $D$ which can be computed in $O(m)$ time [11]. Let $v$ be the vertex of $G$ with coordinates all equal to zero. We order the vertices using a BFS traversal of the graph starting at $v$. If $x y$ is an edge, then it is a down-edge (resp. up-edge, resp. cross-edge) of $x$ when $d(v, x)<d(v, y)$ (resp. $d(v, x)>d(v, y)$, resp. $d(v, x)=d(v, y))$ where $d$ denotes the distance in $G$. We proceed as described in Algorithm 1. We color the edges of $G$ using the prime decomposition $D$ of $G$ : we associate to each factor $X$ of $D$ a color, which is then assigned to every edge belonging to an $X$-layer of $G$. We maintain a temporary decomposition $J$ of $G$ for which we merge some factors, by means of recoloring the edges, during the algorithm. Our goal, at the end of the algorithm, is that $J=P$ where $P$ is the prime $s$-decomposition of $(G, \sigma)$. We note $p_{i}(e)$ the projection of an edge $e=x y \in J_{i}^{x}$ to the temporary $J_{i}$-layer $J_{i}^{v}$.

First note that in Algorithm 1, the set Done is not used. Therefore, it can be omitted. Its only purpose is to ease the correctness analysis of the algorithm. Let us make a few more remarks. The set Done (resp. Treated) is used to record which vertex (resp. edge) has been processed by the algorithm. The set $S$ corresponds to the set of vertices for which we have decided whether they need to be switched or not. If $x \in$ Done at some 
point of the algorithm then all its incident edges belong to the set Treated. Moreover, by construction of the BFS ordering, if $x y$ is a down-edge of $x$ in $J_{i}^{x}$ then, for every vertex $z$, the projection $x^{\prime} y^{\prime}$ of $x y$ on $J_{i}^{z}$ is a down-edge of $x^{\prime}$.

Claim 19. After the merging in line 17 of the algorithm, $v, y$ and $x$ belong to the same layer.

Proof. We just need to prove that $y$ and $v$ belong to the same layer after merging. Note that a layer $J_{i}^{a}$ corresponds to all the vertices $b$ which differ from $a$ only by the $i$ th coordinate (in the current decomposition). Note also that the coordinate vector of a neighbor of $y$ and the coordinate vector of $y$ differ by only one coordinate. For any non-zero coordinate of $y$, there is an up-edge $y z$ of $y$ to a neighbor $z$ of $y$ which differs only on this non-zero coordinate (as the ordering is a BFS ordering and by the Cartesian product structure), therefore all factors $J_{\ell}$ corresponding to non-zero coordinates of $y$ are merged. Hence, in this new coordinate system, $y$ has at most one non-zero coordinate and thus $y$ and $v$ are in the same layer.

Claim 20. Let $a b$ and $a^{\prime} b^{\prime}$ be two edges of the set Treated at any moment of the algorithm. If $a^{\prime} b^{\prime} \in J_{i}^{a^{\prime}}$ for some $i$ and $p_{i}\left(a^{\prime} b^{\prime}\right)=p_{i}(a b)$ (i.e. they represent the same edge of $\left.J_{i}\right)$, then $a b$ and $a^{\prime} b^{\prime}$ have the same sign.

Proof. By contradiction, suppose that $a^{\prime} b^{\prime}$ is the first edge such that, when added to Treated, there exists some edge $a b \in$ Treated such that $p_{i}\left(a^{\prime} b^{\prime}\right)=p_{i}(a b)$ and $a^{\prime} b^{\prime}$ and $a b$ do not have the same sign. Let $a^{\prime \prime} b^{\prime \prime}$ be the edge $p_{i}\left(a^{\prime} b^{\prime}\right)$. Note that no edge in Treated can change sign once it is into the set as both its endpoints are in $S$. By definition of $a^{\prime} b^{\prime}, a b$ and $a^{\prime \prime} b^{\prime \prime}$ have the same sign since they both project to $a^{\prime \prime} b^{\prime \prime}$. Hence, it must be that $a^{\prime} b^{\prime}$ and $a^{\prime \prime} b^{\prime \prime}$ do not have the same sign.

Note that, $a^{\prime} b^{\prime}$ cannot be treated in the third if statement at line 16 , as otherwise it would belong to some layer $J_{i}^{v}$ after merging by Claim 19 and thus $a^{\prime} b^{\prime}$ would project to itself. Since $a^{\prime} b^{\prime}$ went through one of the first two if statements (lines 11 and 14), $a^{\prime} b^{\prime}$ and $a^{\prime \prime} b^{\prime \prime}$ have the same sign, a contradiction.

\section{Proof of Theorem 18.}

Correctness: First, let us show that $J$ is finer than $P$, the prime $s$-decomposition of $(G, \sigma)$, at each step of the algorithm. It is true at the beginning of the algorithm by Theorem 15 as $J=D$. Suppose that $J$ is finer than $P$ at the beginning of step 8 . In the if statement, if we enter the first two cases then we do not change $J$. Hence it is still finer than $P$ at the end of the loop.

Suppose $x y$ and $x^{\prime} y^{\prime}$ are not of the same sign and $y \in S$ (i.e. we enter line 17). As $y \in S$ and $x y \notin$ Treated, there is some neighbor $z$ of $y$ for which $z \in$ Done. We consider two cases depending on whether $z \in J_{i}^{x}$ or $z \notin J_{i}^{x}$.

Suppose first that $z \in J_{i}^{x}$.

Take a shortest path $P_{z}$ in $J_{i}^{x}$ from $z$ to the projection $p_{v}$ of $v$ on $J_{i}^{x}$. All vertices of the path appear before $z$ in the BFS ordering, thus all the edges of the path belong to the set Treated. The same holds for a shortest path $P_{x}$ from $p_{v}$ to $x$. In particular the walk $W$ obtained by concatenating $y z, P_{z}$ and $P_{x}$ has all its edges in Treated. This implies that $W$ and $W^{\prime}$, its projection on $J_{i}^{v}$, have the same sign by Claim 20. Hence the closed 


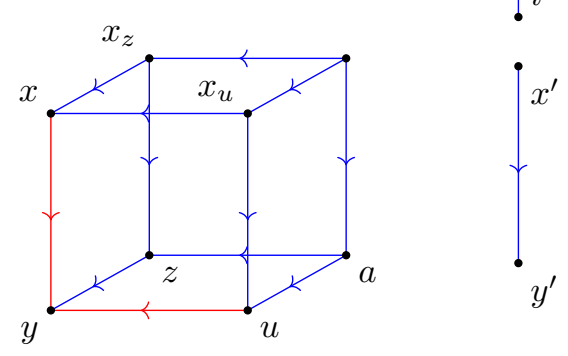

Figure 2: The second case of the correctness analysis. For simplicity, all edges which are in Treated are positive. The orientation of the edges represents the BFS order. The neighbors of $v$ are labelled with the temporary color of their edge with $v$.

walk $C$ obtained by concatenating $W$ with $x y$ and its projection ( $W^{\prime}$ concatenated with $\left.x^{\prime} y^{\prime}\right)$ have different signs and $J_{i}^{v}$ and $J_{i}^{x}$ do not have the same signature.

Let $u$ be a neighbor of $y$ such that $u y$ is an up-edge of $y$ and $u \notin J_{i}^{x}$. Every edge $e^{\prime}$ of the projection $C^{\prime}$ of $C$ on $J_{i}^{u}$ is in Treated as $d\left(v, e^{\prime}\right)<d(v, e)$ where $e$ is the counterpart of $e^{\prime}$ in $C$ (all vertices of $C$ have an up-edge to their projection on $J_{i}^{u}$ ). In particular $C$ and $C^{\prime}$ do not have the same sign and $J_{i}^{x}$ and $J_{i}^{u}$ do not have the same signature.

This implies that both layers are in the same factor of $P$. Indeed suppose that this is not the case. Then all cycles $a b b^{\prime} a^{\prime}$, such that $a b \in J_{i}^{x}$ and $a^{\prime} b^{\prime}$ is its projection on $J_{i}^{u}$, must be $B C_{4}$. For all edges $a b$ of $W, a b$ and $a^{\prime} b^{\prime}$ have the same sign by Claim 20, hence $a a^{\prime}$ and $b b^{\prime}$ also have the same sign (since the cycle is balanced). Now let $x^{\prime \prime} y^{\prime \prime}$ be the projection of $x y$ on $J_{i}^{u}$. By going around $W$ and by the previous observation, $x x^{\prime \prime}$ and $y y^{\prime \prime}$ have the same sign. Note that $x y$ and $x^{\prime \prime} y^{\prime \prime}$ do not have the same sign as $x^{\prime \prime} y^{\prime \prime} \in$ Treated $\left(x^{\prime \prime} y^{\prime \prime}\right.$ has the same sign as $\left.x^{\prime} y^{\prime}\right)$. This implies that $x y y^{\prime \prime} x^{\prime \prime}$ is a $U C_{4}$, a contradiction.

Hence we need to merge all temporary colors of all up-edges of $y$ (including color $i$ ). Thus after this step $J$ is still finer than $P$.

Suppose now that $z \notin J_{i}^{x}$ (see Figure 2).

In this case, $z$ is the projection of $y$ on $J_{i}^{z}$. Let $x_{z}$ be the projection of $x$ on $J_{i}^{z}$. Since $y z$ is an up-egde of $y, x x_{z}$ is an up-edge of $x$ and $x_{z} \in$ Done. Note that $x x_{z}$ and $y z$ have the same sign since both are in Treated. Also note that $x_{z} z$ and $x^{\prime} y^{\prime}$ have the same sign since $x_{z} z \in$ Treated. Hence $x y z x_{w}$ is a $U C_{4}$. By the same arguments as before, these four vertices belong to the same signed factor of $(G, \sigma)$, hence we must merge $i$ and, say $j$, the temporary colors of $x y$ and $y z$ respectively.

Let $u$ be a neighbor of $y$ such that $u y$ is an up-edge of $y$ of temporary color $k \notin\{i, j\}$. Let $x_{u}$ be the projection of $x$ on $J_{i}^{u}$. Note that $x_{u} u$ and $x^{\prime} y^{\prime}$ have the same sign as $d\left(x_{u}, v\right)<d(x, v)$ (i.e. $x_{u} \in$ Done). If $x x_{u}$ and $y u$ have the same sign, we have a $U C_{4}$ and must merge the temporary colors $i$ and $k$. Suppose they have different signs. Note that $y$ and $z$ (resp. $u$ ) differ only by their $j$ th coordinate (resp. $k$ th coordinate). Let $a$ be the vertex with the same coordinate as $u$ except for its $k$ th coordinate which is equal to the $k$ th coordinate of $z$ (see Figure 2). Note that $a$ appears before $z$ and $u$ in the 
BFS ordering. Since the vertex $a$ is a neighbor of $z$ and $u$, both edges $z a$ and $u a$ are down-edges of $a$. Hence $z a \in$ Treated and $z a$ has the same sign as $x x_{u}$ which is different from the sign of $u y$, and $y z$ and $u a$ also have the same sign since both are in Treated. In particular yuaz is a $U C_{4}$ and these four vertices must be in the same factor of $P$. This implies that we must merge the temporary colors $j$ and $k$ which implies merging $i$ and $k$.

At the end $J$ is finer than $P$ and $J$ is an $s$-decomposition by Claim 20. Hence $J=P$.

Complexity: Due to the similarity of our algorithm with the one in [12], most of the complexity arguments given in [12] are still valid for our algorithm. The only differences between the two algorithms are the presence of the three sets Done, $S$ and Treated, two more if blocks and the need to switch at some vertices. Let us address these three points. Each set can be encoded by a boolean in the data structure of vertices/edges. The second for loop checks each edge $x y$ twice, once for each endpoint, but this still amounts to a $O(m)$ iteration of the loop. The two additional if blocks are a $O(1)$ overhead for each iteration of the loop. The switch operation is another $O(m)$ total overhead as each edge can be switched at most once thanks to the presence of the set $S$. Hence the algorithm runs in time $O(m)$. The reader can find more details in [12], and in particular, how to compute the projections in constant time.

Note that this algorithm not only computes the prime $s$-decomposition of $(G, \sigma)$ but finds a signature $\sigma^{\prime} \equiv \sigma$ for which all layers of the Cartesian products have the same signature as their corresponding factors.

\section{Chromatic number of Cartesian products of complete signed graphs and upper bounds}

In this section, we show a simple upper bound on the chromatic number of a Cartesian product of two signed graphs and compute the chromatic number of some special complete signed graphs. We start by defining a useful tool on signed graphs.

\section{1. s-redundant sets}

In what follows we define the notion of an $s$-redundant set in a signed graph. Intuitively, if $S$ is an $s$-redundant set of $(G, \sigma)$ and $x$ and $y$ are two vertices cannot be mapped to a same vertex by any homomorphism of $(G, \sigma)$, then they cannot be mapped to a same vertex by a homomorphism of $(G, \sigma)-S$.

Definition 21. Let $(G, \sigma)$ be a signed graph and $S \subseteq V(G)$. We say that the set $S$ is $s$-redundant if and only if, for every $x, y \in V(G)-S$ such that $x y \notin E(G)$, every $z \in S$ and every signature $\sigma^{\prime}$ with $\sigma^{\prime} \equiv \sigma$, if $x z y=U P_{3}$ in $\left(G, \sigma^{\prime}\right)$ then there exists $w \in V(G)-S$ such that $x w y=U P_{3}$ in $\left(G, \sigma^{\prime}\right)$.

The following proposition provides an alternative formulation of the definition which is useful in order to prove that a set is an $s$-redundant set.

Proposition 22. If $(G, \sigma)$ is a signed graph and $S \subseteq V(G)$, then $S$ is s-redundant if and only if for every $z \in S$, and every $x, y \in N(z) \backslash S$ with $x y \notin E(G)$, there exists $w \in V(G) \backslash S$ such that $x w y z$ is a $B C_{4}$. 
Proof. Take $x, y \in V(G)-S$ such that $x y \notin E(G)$ and $z \in S$. If $x z y=U P_{3}$ in a signature $\sigma^{\prime} \equiv \sigma$, then $x, y \in N(z)$. Now if $S$ is an $s$-redundant set, then with the notation of the definition $x z y w$ is a $B C_{4}$ in $\left(G, \sigma^{\prime}\right)$ and thus in $(G, \sigma)$. If $x z y w$ is a $B C_{4}$ and $x z y$ is a $U P_{3}$ in a given signature $\sigma^{\prime}$, then $x w y$ is also a $U P_{3}$ as $x z y w$ is balanced. This proves the equivalence between the two statements.

The next theorem is the reason why we defined this notion. It allows us to compute an upper bound on the chromatic number of a signed graph as a function of the chromatic number of one of its subgraphs. One example of utilisation of this notion is given by the proof of Theorem 25.

Theorem 23. If $(G, \sigma)$ is a signed graph and $S$ is an s-redundant set of $(G, \sigma)$, then

$$
\chi_{s}(G, \sigma) \leq|S|+\chi_{s}((G, \sigma)-S) .
$$

Proof. Let $c$ be a coloring of a signed graph $\left(G, \sigma^{\prime}\right)-S$ with $\chi_{s}((G, \sigma)-S)$ colors where $\left(G, \sigma^{\prime}\right) \equiv(G, \sigma)$. We define the coloring $c^{\prime}$ of $\left(G, \sigma^{\prime}\right)$ as follows: $c^{\prime}(v)=c(v)$ when $v \notin S$ and $c^{\prime}(v)$ is a new color when $v \in S$. Hence $c^{\prime}$ uses at most $|S|+\chi_{s}((G, \sigma)-S)$ colors.

It is left to show that it is indeed a coloring of $\left(G, \sigma^{\prime}\right)$. As $c$ is a coloring, $c^{\prime}$ does not assign the same color to two adjacent vertices. Suppose, by contradiction, that there exists two edges $x y$ and $x^{\prime} y^{\prime}$ of opposite sign such that $c^{\prime}(x)=c^{\prime}\left(x^{\prime}\right)$ and $c^{\prime}(y)=c^{\prime}\left(y^{\prime}\right)$. As $c$ is a coloring, all four vertices cannot be in $G-S$. W.l.o.g. suppose that $x \in S$. By definition of $c^{\prime}, x^{\prime}=x, y, y^{\prime} \notin S$ and $y x y^{\prime}$ is a $U P_{3}$ in $\left(G, \sigma^{\prime}\right)$. As $S$ is an $s$-redundant set, there exists $w \notin S$ such that $y w y^{\prime}$ is a $U P_{3}$ in $\left(G, \sigma^{\prime}\right)-S$. This contradicts the fact that $c$ is a coloring of $\left(G, \sigma^{\prime}\right)-S$.

This result does not hold for any set $S$. For example, if $(G, \sigma)=U C_{4}$ and $S=\{v\}$ is a single vertex of $G$, then $\chi_{s}(G, \sigma)=4$ and $\chi_{s}((G, \sigma)-v)=2$.

\subsection{Back to Cartesian products of complete signed graphs}

As a direct corollary of Theorem 8 , we get the following upper bound on the chromatic number of a Cartesian product of signed graphs.

Corollary 24. If $\left(G_{1}, \sigma_{1}\right), \ldots,\left(G_{k}, \sigma_{k}\right)$ are $k$ signed graphs, then:

$$
\chi_{s}\left(\left(G_{1}, \sigma_{1}\right) \square \cdots \square\left(G_{k}, \sigma_{k}\right)\right) \leq \prod_{1 \leq i \leq k} \chi_{s}\left(G_{i}, \sigma_{i}\right) .
$$

We consider the Cartesian product of balanced and antibalanced complete graphs in our next result. Recall that $K_{p}^{+}$(resp. $K_{q}^{-}$) is the complete graph with only positive edges (resp. negative edges).

Theorem 25. For every two integers $p, q$ with $p, q \geq 2$, we have

$$
\chi_{s}\left(K_{p}^{+} \square K_{q}^{-}\right)=\left\lceil\frac{p q}{2}\right\rceil .
$$




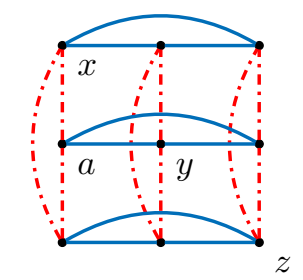

(a) Notation of the proof

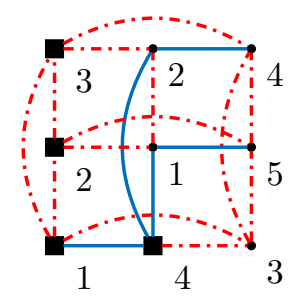

(b) A coloring of $(H, \sigma)$ with 5 colors

Figure 3: The signed graph $(H, \sigma)=K_{3}^{+} \square K_{3}^{-}$of Theorem 25. The big squared vertices have been switched.

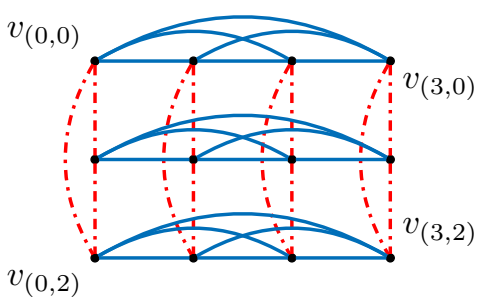

(a) The signed graph $(P, \pi)$

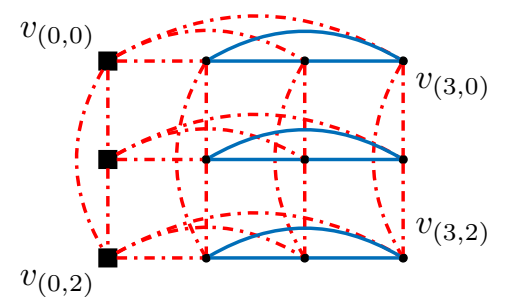

(b) The signed graph $\left(P, \pi^{\prime}\right)$ where the big squared vertices have been switched.

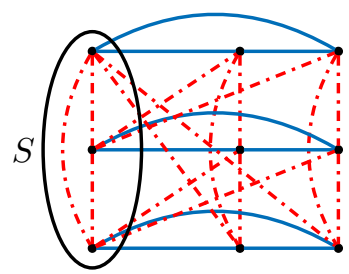

(c) The signed graph $\left(P^{\prime \prime}, \pi^{\prime \prime}\right)$ with the set $S$.

Figure 4: The signed graphs $(P, \pi),\left(P, \pi^{\prime}\right)$ and $\left(P^{\prime \prime}, \pi^{\prime \prime}\right)$ of Theorem 25 when $(P, \pi)=K_{4}^{+} \square K_{3}^{-}$.

Proof. Let us note $(P, \pi)=K_{p}^{+} \square K_{q}^{-}$. By symmetry between the sets of positive and negative edges, we can suppose $p \geq q$. First let us show that $\chi_{s}(P, \pi) \geq\left\lceil\frac{p q}{2}\right\rceil$.

Suppose it is not the case. Let $\varphi$ be an optimal homomorphism of $(P, \pi)$. By the pigeon hole principle, there exist $x, y$ and $z$ three vertices of the Cartesian product with the same image by $\varphi$. They belong to three distinct $K_{p}^{+}$-layers and three distinct $K_{q}^{-}$layer as these are complete graphs. Consider the subgraph $(H, \sigma)$ of $(P, \pi)$ composed of vertices which are in the same $K_{p}^{+}$-layers as one of $x, y, z$ and in the same $K_{q}^{+}$-layers as one of $x, y$ and $z$. We have $(H, \sigma)=K_{3}^{+} \square K_{3}^{-}$(see Figure 3).

By assumption $x, y$ and $z$ of $(H, \sigma)$ are identified by $\varphi$ (possibly after switching some of them). By the pigeon hole principle, two of $x, y$ and $z$ are both switched or both non-switched. Without loss of generality suppose they are $x$ and $y$. Then if $a$ is one of their common neighbors in $H$, the edges $x a$ and $y a$ are of different signs, thus $x$ and $y$ cannot be identified. This is a contradiction. 
We now prove that $\chi_{s}(P, \pi) \leq\left\lceil\frac{p q}{2}\right\rceil$ by induction. If $p=2$, then $(P, \pi) \equiv B C_{4}$ and $\chi_{s}(P, \pi)=2 \leq 2$. If $p=3$ and $q=2$, then $(P, \pi) \equiv B C_{3} \square K_{2}$ whose chromatic number is 3. If $p=3$ and $q=3$, then $(P, \pi) \equiv K_{3}^{+} \square K_{3}^{-}$. In this case, we have $\chi_{s}(P, \pi)=5$, as Figure 3 gives a 5 -coloring of $(P, \pi)$.

Now we can assume $p \geq 4$. Let $V(P)=\left\{v_{(i, j)}, 0 \leq i<p, 0 \leq j<q\right\}$ such that for every $i$, the set $\left\{v_{(i, j)}\right\}_{0 \leq j<q}$ induces a negative complete graph and for every $j$, the set $\left\{v_{(i, j)}\right\}_{0 \leq i<p}$ induces a positive complete graph (see Figure $4 \mathrm{a}$ ). Now switch all vertices in $\left\{v_{(i, j)} \mid i=0\right\}$ to obtain the signed graph $\left(P, \pi^{\prime}\right)$ (see Figure $4 \mathrm{~b}$ ) and then identify $v_{(0, j)}$ with $v_{(1, j+1)}$ (which are non adjacent) for every $j \in \llbracket 0, q-1 \rrbracket$, where indices are taken modulo $q$, to obtain the graph $\left(P^{\prime \prime}, \pi^{\prime \prime}\right)$ (see Figure 4 c). Let $S$ be the set of identified vertices in $\left(P^{\prime}, \pi^{\prime}\right)$. We want to show that $S$ is $s$-redundant in order to use the induction hypothesis. Take $z \in S$ and $x, y \in N(z) \backslash S$ such that $x y \notin E\left(P^{\prime \prime}\right)$. If $x z y$ is an unbalanced path of length 2 , then $x$ is some $v_{(i, j)}$ and $y$ is some $v_{(k, j+1)}$ with $i, k \geq 2$. For $a=v_{(i, j+1)}, x a y z$ is a $B C_{4}$.

By Proposition 22, $S$ is $s$-redundant and thus

$$
\chi_{s}(P, \pi) \leq \chi_{s}\left(P^{\prime \prime}, \pi^{\prime \prime}\right) \leq|S|+\chi_{s}\left(\left(P^{\prime}, \pi^{\prime}\right)-S\right)
$$

by Theorem 23. By induction hypothesis, as $\left(P^{\prime \prime}, \pi^{\prime \prime}\right)-S=K_{p-2}^{+} \square K_{q}^{-}$, we get $\chi_{s}\left(\left(P^{\prime \prime}, \pi^{\prime \prime}\right)-S\right) \leq\left\lceil\frac{(p-2) q}{2}\right\rceil$. Thus $\chi_{s}(P, \pi) \leq q+\left\lceil\frac{p q}{2}\right\rceil-q \leq\left\lceil\frac{p q}{2}\right\rceil$.

Note that $\chi_{s}\left(K_{p}^{+} \square K_{p}^{-}\right)=O\left(\Delta^{2}\right)$ where $\Delta$ is the maximum degree of $K_{p}^{+} \square K_{p}^{-}$(i.e. $\Delta=2 p-1)$. Indeed, the chromatic number is $O\left(p^{2}\right)$ while $\Delta^{2}=(2 p-1)^{2}=4 p^{2}-4 p+1$. Also, for this Cartesian product, the upper bound of Corollary 24 is $p^{2}$ while we proved in Theorem 25 that the chromatic number is $\left\lceil\frac{p^{2}}{2}\right\rceil$. We thus have an example where the chromatic number is greater than half the simple upper bound.

Question 26. What is the supremum of the set of real numbers $\lambda \in\left[\frac{1}{2}, 1\right]$ such that there exist signed graphs $\left(G_{1}, \sigma_{1}\right), \ldots,\left(G_{k}, \sigma_{k}\right)$, each with at least one edge, such that:

$$
\chi_{s}\left(\left(G_{1}, \sigma_{1}\right) \square \cdots \square\left(G_{k}, \sigma_{k}\right)\right) \leq \lambda \prod_{1 \leq i \leq k} \chi_{s}\left(G_{i}, \sigma_{i}\right) ?
$$

In Figure 5, we have an example of a graph $K$ such that $K \square K_{2}$ has chromatic number 25 (checked by computer). The ratio between the chromatic number and the upper bound is $\frac{25}{36}=0.69444$. It is the largest ratio we have found by randomly sampling bigger and bigger complete signed graphs. This leads us to believe that the following conjecture holds.

Conjecture 27. For every fixed $\varepsilon>0$, there exist signed graphs $\left(G_{1}, \sigma_{1}\right), \ldots,\left(G_{k}, \sigma_{k}\right)$, with each at least one edge, such that:

$$
\chi_{s}\left(\left(G_{1}, \sigma_{1}\right) \square \cdots \square\left(G_{k}, \sigma_{k}\right)\right) \geq(1-\varepsilon) \cdot \prod_{1 \leq i \leq k} \chi_{s}\left(G_{i}, \sigma_{i}\right) .
$$




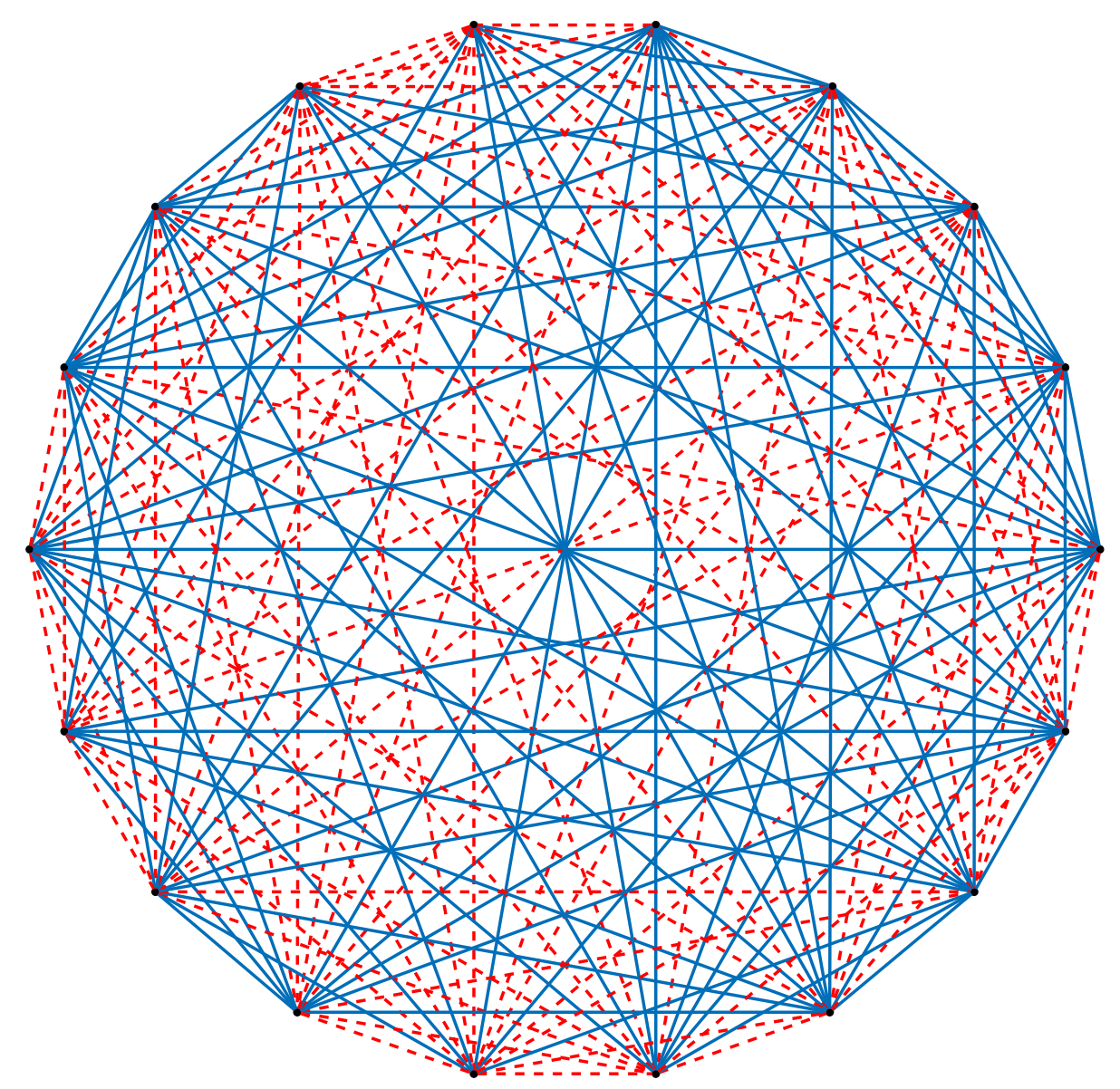

Figure 5: A signed graph $K$ of order 18 such that $\chi_{s}\left(K \square K_{2}\right)=25$.

\section{Chromatic number of Cartesian products of signed cycles}

The goal of this section is to determine the chromatic number of the Cartesian product of two signed cycles. As there are four kind of cycles (balanced/unbalanced and even/odd length), we have a number of cases to analyse. In most cases some simple observations are sufficient to conclude. For the other cases, we need the following lemma whose proof is given in subsections 6.1 to 6.6 , due to its length.

Lemma 28. For every two integers $p, q \in \mathbb{N}$ :

$$
\chi_{s}\left(U C_{q} \square B C_{2 p+1}\right)>4 .
$$

With this lemma, we can state the main result of this section.

Theorem 29. If $\left(C_{1}, \sigma\right)$ and $\left(C_{2}, \sigma_{2}\right)$ are two signed cycles, then the chromatic number of $(P, \pi)=\left(C_{1}, \sigma_{1}\right) \square\left(C_{2}, \sigma_{2}\right)$ is given by Table 1, depending on the types of $\left(C_{1}, \sigma_{1}\right)$ and $\left(C_{2}, \sigma_{2}\right)$. 


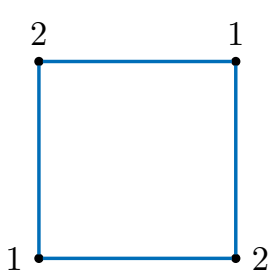

(a) $\chi_{s}\left(K_{2} \square K_{2}\right) \leq 2$

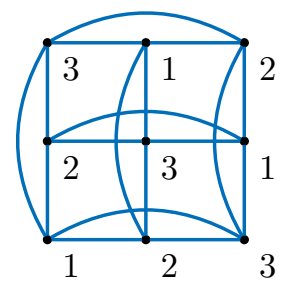

(d) $\chi_{s}\left(B C_{3} \square B C_{3}\right) \leq$ 3

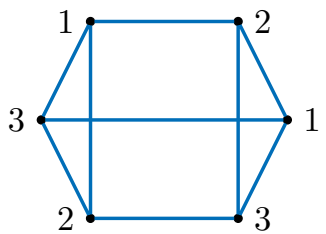

(b) $\chi_{s}\left(B C_{3} \square K_{2}\right) \leq 3$

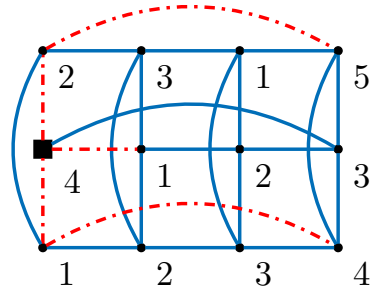

(e) $\chi_{s}\left(B C_{3} \square U C_{4}\right) \leq 5$

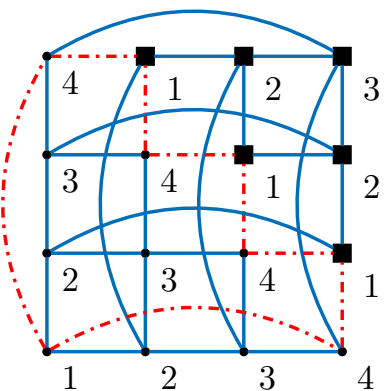

(g) $\chi_{s}\left(U C_{4} \square U C_{4}\right) \leq 4$

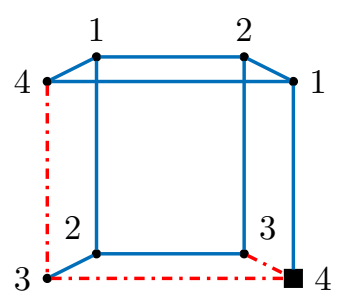

(c) $\chi_{s}\left(U C_{4} \square K_{2}\right) \leq 4$

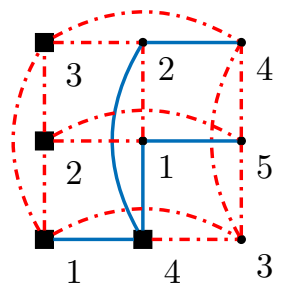

(f) $\chi_{s}\left(U C_{3} \square B C_{3}\right) \leq 5$ in the Cartesian product.

\begin{tabular}{|l|l|l|l|l|}
\hline$\left(C_{1}, \sigma_{1}\right) \square\left(C_{2}, \sigma_{2}\right)$ & $B C_{\text {even }}$ & $B C_{\text {odd }}$ & $U C_{\text {even }}$ & $U C_{\text {odd }}$ \\
\hline$B C_{\text {even }}$ & 2 & 3 & 4 & 3 \\
\hline$B C_{\text {odd }}$ & 3 & 3 & 5 & 5 \\
\hline$U C_{\text {even }}$ & 4 & 5 & 4 & 5 \\
\hline$U C_{\text {odd }}$ & 3 & 5 & 5 & 3 \\
\hline
\end{tabular}

Table 1: The chromatic number of Cartesian products of signed cycles.

Proof. If $G$ is a cycle of type $B C_{\text {even }}$ (resp. $B C_{o d d}, U C_{\text {even }}, U C_{\text {odd }}$ ), then $G \longrightarrow_{s} B C_{2}=$ $K_{2}$ (resp. $B C_{3}, U C_{4}, U C_{3}$ ). By computing the chromatic numbers of the Cartesian products of $(G, \sigma)$ and $(H, \pi)$ when they belong to $\left\{K_{2}, B C_{3}, U C_{4}, U C_{3}\right\}$, we get an upper bound for each of the Cartesian product type equal to the corresponding value 


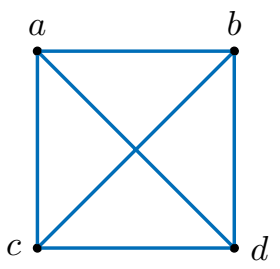

(a) $K_{4}^{+}$

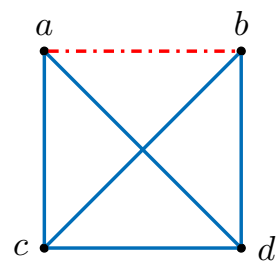

(b) $K_{4}^{\text {mixed }}$

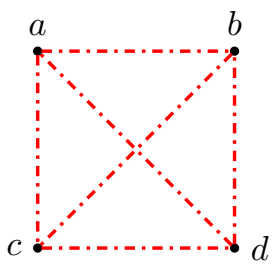

(c) $K_{4}^{-}$

Figure 7: The three complete signed graphs of order 4

in the table. These cases, up to symmetry between the sets of positives and negatives edges, are represented in Figure 6. Note that to color some graphs, we switched some vertices.

For the lower bound, note that $\chi_{s}\left(\left(C_{1}, \sigma_{1}\right) \square\left(C_{2}, \sigma_{2}\right)\right) \geq \max \left(\chi_{s}\left(C_{1}, \sigma_{1}\right), \chi_{s}\left(C_{2}, \sigma_{2}\right)\right)$. Theorem 2 concludes for the cases where the chromatic number is at most 4 . Lemma 28 allows us to conclude for the remaining cases as $\chi_{s}\left(U C_{q} \square B C_{2 p+1}\right)=\chi_{s}\left(U C_{q} \square U C_{2 p+1}\right)$ by symmetry between the two edge types.

One further question would be to compute the chromatic number of the Cartesian product of an arbitrary number of signed cycles. Note that $B C_{3} \square B C_{3} \longrightarrow_{s} B C_{3}$, and that the same holds for $K_{2}, U C_{3}$ and $U C_{4}$. This implies that, for these four graphs, it is only interesting to look at Cartesian products of the form $K_{2}^{a} \square B C_{3}^{b} \square U C_{4}^{c} \square U C_{3}^{d}$ where $a, b, c, d \in\{0,1\}$. Moreover, we can suppose that $a=0$ if one of $b, c$ or $d$ is non zero. Thus the only interesting case left to solve is $\chi_{s}\left(B C_{3} \square U C_{3} \square U C_{4}\right)$.

To extend this to any length, using the same argument as in Theorem 29, would require that we obtain a lower bound for $\chi_{s}\left(B C_{2 p+1} \square U C_{2 q+1} \square U C_{2 r}\right)$ equal to $\chi_{s}\left(B C_{3} \square U C_{3} \square U C_{4}\right)$.

\subsection{Definitions and preliminary results}

We start by recalling some more definitions.

A graph $G$ is bipartite if we can partition $V(G)$ into $A \uplus B$ (where $\uplus$ is the disjoint union) such that every edge $x y$ of $G$ has one endpoint in $A$ and one endpoint in $B$.

For a group $(H,+, 0)$, noted simply $H$, and a subgroup $Q$ of $H$, the quotient $H / Q$ is the group $(\{\bar{x} \mid x \in H\},+, \overline{0})$ where $\bar{x}=\{y \in H \mid y=x+q, q \in Q\}$ is the equivalence class of $x$ and where the + operation verifies $\bar{x}+\bar{y}=\overline{x+y}$. If $G$ is a graph with vertex set a group $H$ and $Q$ is a subgroup of $H$, then the quotient graph $G / Q$ over the vertices $H / Q$ is defined by identifying the vertices in the same equivalence class. Similarly, if $W=s_{0}, \ldots, s_{n}$ is a walk on $G$, then the quotient walk $W^{\prime}$ on $G / Q$ is the sequence $\overline{s_{0}}, \ldots, \overline{s_{n}}$.

Now, we count the number of signed complete graphs on four vertices. This result will be useful in the proof of Lemma 28 .

Theorem 30. There are three complete signed graphs of order 4 (see Figure 7). They are the signed graph $K_{4}^{+}=\left(K_{4}, \varnothing\right)$ with only positive edges, the signed graph $K_{4}^{-}=$ 
$\left(K_{4}, E\left(K_{4}\right)\right)$ with only negative edges and the signed graph $K_{4}^{\text {mixed }}=\left(K_{4},\{a b\}\right)$ where a and $b$ are two vertices of $K_{4}$.

Proof. Let $\left(K_{4}, \sigma\right)$ be a complete signed graph on four vertices. Arbitrarily choose $u$ to be one of the vertices of $\left(K_{4}, \sigma\right)$. By switching the neighbors of $u$ if needed, we can suppose that $u$ is only incident to positive edges. Let $x, y, z$ be the other three vertices of $\left(K_{4}, \sigma\right)$. If the triangle $x y z$ is all positive, then $\left(K_{4}, \sigma\right)=K_{4}^{+}$, if the triangle is all negative, then by switching $u$, we get $\left(K_{4}, \sigma\right)=K_{4}^{-}$. If the triangle has only one negative edge, then $\left(K_{4}, \sigma\right)=K_{4}^{\text {mixed }}$. Otherwise, the triangle has two negative edges, by switching the vertex with the two negative edges, we get $\left(K_{4}, \sigma\right)=K_{4}^{\text {mixed }}$.

\subsection{Beginning of the proof of Lemma 28}

Our goal is to prove Lemma 28. For that, take some integers $p$ and $q$, let $(P, \pi)=$ $U C_{q} \square B C_{2 p+1}$, and suppose that, by absurd, $\chi_{s}(P, \pi) \leq 4$.

Claim 31. We have $(P, \pi) \longrightarrow_{s} K_{4}^{\text {mixed }}$.

Proof. Since $\chi_{s}(P, \pi) \leq 4,(P, \pi) \longrightarrow_{s}\left(K_{4}, \rho\right)$ for some signature $\rho$ of $K_{4}$.

Every equivalent signature of $B C_{2 p+1}$ has at least one positive edge. Similarly, every equivalent signature of $U C_{q}$ has at least one negative edge. Thus, in every equivalent signature of $(P, \pi)$, there is at least one positive edge and one negative edge. So $(H, \rho)$ cannot be $\left(K_{4}, \varnothing\right)$ nor $\left(K_{4}, E\left(K_{4}\right)\right)$. By Theorem 30 , since there are only three complete signed graphs of order $4,(H, \rho)$ is $K_{4}^{\text {mixed }}$.

From now on, we suppose that we fixed a homomorphism $\varphi$ of $(P, \pi)$ to $K_{4}^{\text {mixed }}$. We label the vertices of $K_{4}^{\text {mixed }}$ as in Figure $7 \mathrm{~b}$. Therefore, there exists a signed graph $\left(P, \pi^{\prime}\right) \equiv(P, \pi)$ for which $v \mapsto \varphi(v)$ is a coloring.

The proof of Lemma 28 is divided into four parts. First, by considering the graph $P$ as a toroidal grid, we define what we mean for a walk to make a "turn" around the torus in subsection 6.3. Then, by considering the coloring of $\left(P, \pi^{\prime}\right)$ corresponding to $\varphi$ and the connected components of $\left(P, \pi^{\prime}\right)$ induced by colors $a$ and $b$, we link the number of "crossings" of some boundaries of the components with a vertical (or horizontal) cycle and the number of $a b$ edges of this cycle in subsection 6.4. In subsection 6.5, we connect this number of "crossings" to the number of turns and we conclude the proof in subsection 6.6.

\subsection{Number of turns in $P$}

The goal of this subsection is twofold. First, we want to establish another definition of $P$ as a toroidal grid i.e. the quotient of some infinite grid. Secondly, we want to define the quantities $\tau_{x}(W)$ and $\tau_{y}(W)$ for each closed walk $W$ of $T$. They represent the number of turns in each direction of the torus made by the closed walk $W$.

Definition 32. We can associate with $\mathbb{Z}^{2}$ an infinite graph $G^{\infty}$ whose vertex set $V\left(G^{\infty}\right)$ is the set $\left\{v_{x, y} \mid(x, y) \in \mathbb{Z}^{2}\right\}$ and whose edge set is the set of pairs $\left\{v_{x, y} v_{x^{\prime}, y^{\prime}}\right\}$, where either $x=x^{\prime}$ and $\left|y-y^{\prime}\right|=1$, or $y=y^{\prime}$ and $\left|x-x^{\prime}\right|=1$. We can then redefine the graph $P$ as the quotient $G^{\infty} / Q$ where $Q=\mathbb{Z}_{2 p+1} \times \mathbb{Z}_{q}$. In other words take the graph $G^{\infty}$ where we identify each vertex $v_{x, y}$ with $v_{x^{\prime}, y^{\prime}}$ when $x-x^{\prime}$ is a multiple of $2 p+1$ and $y-y^{\prime}$ is a multiple of $q$. The graph $G^{\infty}$ can be seen as an unfolding of the toroidal grid $P$. Figure 8 represents a subgraph of $G^{\infty}$ when $q=4$ and $2 p+1=3$. An edge of $G^{\infty}$ 


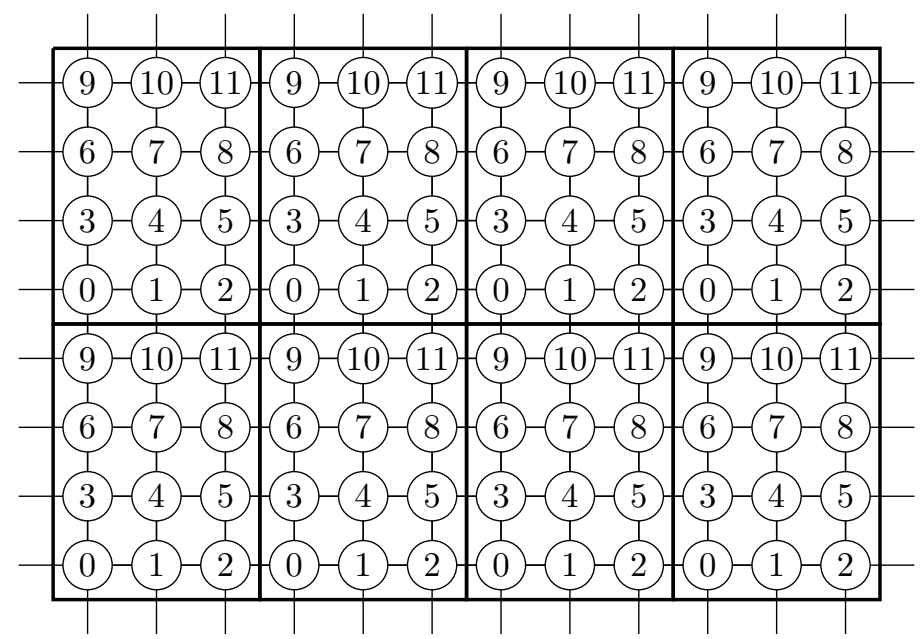

Figure 8: A subgraph of the graph $G$. Vertices with the same label are identified in $P$. Here $q=4$ and $2 p+1=3$.

of the form $v_{u, w} v_{u+i, w}$ (resp. $v_{u, w} v_{u, w+i}$ ) for $i \in\{-1,1\}$, is an horizontal (resp. vertical) edge of $G^{\infty}$. An edge $e$ of $P$ is an horizontal (resp. vertical) edge if it is the quotient of horizontal (resp. vertical) edges of $G^{\infty}$.

Definition 33. Let $W_{G^{\infty}}$ be a walk in $G^{\infty}$ and $W_{P}$ a walk in $P$. We say that $W_{G^{\infty}}$ is a representation of $W_{P}$ if and only if $W_{G^{\infty}} / Q=W_{P}$. We also say that $W_{G^{\infty}}$ represents $W_{P}$.

By definition, all representations of $W_{P}$ have the same number of vertices as $W_{P}$. Let us make the following observation on the representations of a walk $W_{P}$.

Observation 34. If $W_{G^{\infty}}^{1}=\left(s_{i}^{1}\right)_{0 \leq i \leq n}$ and $W_{G^{\infty}}^{2}=\left(s_{i}^{2}\right)_{0 \leq i \leq n}$ are two walks (of the same length) in $G^{\infty}$ representing $W_{P}$, then there exist $\alpha, \beta \in \mathbb{Z}$ such that for all $i \in\{0, \ldots, n\}$, if $s_{i}^{1}=v_{x, y}$, then $s_{i}^{2}=v_{x+\alpha(2 p+1), y+\beta q}$. In particular, if they have the same starting vertices, then $W_{G^{\infty}}^{1}=W_{G^{\infty}}^{2}$.

We are now ready to define what is a turn of a walk around the torus.

Definition 35. Let $W_{G^{\infty}}$ be a walk in $G^{\infty}$ starting with $v_{x, y}$ and ending with $v_{z, t}$. We define the number of horizontal turns $\tau_{x}$ and the number of vertical turns $\tau_{y}$ of $W_{G^{\infty}}$ by:

$$
\tau_{x}\left(W_{G^{\infty}}\right)=\left|\frac{z-x}{2 p+1}\right|, \tau_{y}\left(W_{G^{\infty}}\right)=\left|\frac{t-y}{q}\right| .
$$

For a closed walk $W_{P}$ in $P$, let $\tau_{x}\left(W_{P}\right)=\tau_{x}\left(W_{G^{\infty}}\right)\left(\operatorname{resp} . \tau_{y}\left(W_{P}\right)=\tau_{y}\left(W_{G^{\infty}}\right)\right)$ be the number of horizontal (resp. vertical) turns of $W_{p}$ where $W_{G \infty}$ is an arbitrary representation of $W_{P}$.

Claim 36. The two quantities $\tau_{x}\left(W_{P}\right)$ and $\tau_{y}\left(W_{P}\right)$ are integers and do not depend on the choice of the representation $W_{G^{\infty}}$ of $W_{P}$. 
Proof. First if $W_{P}$ is a closed walk in $P$ and $W_{G^{\infty}}$ represents $W_{P}$, then $\overline{v_{x, y}}=\overline{v_{z, t}}$ thus $z=x+n(2 p+1)$ and $t=y+m q$ for some integers $n, m \in \mathbb{Z}$. Hence $\tau_{x}\left(W_{G^{\infty}}\right)$ and $\tau_{y}\left(W_{G \infty}\right)$ are integers.

Now take two representations $W_{G^{\infty}}^{1}$ and $W_{G^{\infty}}^{2}$ of $W_{P}$. By Observation 34 , if $W_{G^{\infty}}^{1}$ starts at $v_{x_{1}, y_{1}}$ and ends at $v_{z_{1}, t_{1}}$ while $W_{G \infty}^{2}$ starts at $v_{x_{2}, y_{2}}$ and ends at $v_{z_{2}, t_{2}}$, then $x_{2}=x_{1}+\alpha(2 p+1), y_{2}=y_{1}+\beta q, z_{2}=z_{1}+\alpha(2 p+1)$ and $t_{2}=t_{1}+\beta q$. Thus $\tau_{x}\left(W_{G^{\infty}}^{1}\right)=\tau_{x}\left(W_{G^{\infty}}^{2}\right)$ and $\tau_{y}\left(W_{G^{\infty}}^{1}\right)=\tau_{y}\left(W_{G^{\infty}}^{2}\right)$. Hence this quantity is well defined for $W_{P}$.

The main result of this subsection is the following proposition.

Proposition 37. If $W_{P}$ is a closed walk in $P$ of even length, then:

$$
q \tau_{y}\left(W_{P}\right)+\tau_{x}\left(W_{P}\right) \equiv 0 \quad(\bmod 2) .
$$

Proof. Let $W_{G^{\infty}}$ be a representation of $W_{P}$ in $G^{\infty}$ starting at $v_{x, y}$ and ending at $v_{z, t}$. For each horizontal (resp. vertical) edge $e$ of the form $v_{u, w} v_{u+i, w}$ (resp. $v_{u, w} v_{u, w+i}$ ) for $i \in\{-1,1\}$, let $\ell(e)=i$. Let $E_{h}\left(W_{G^{\infty}}\right)$ be the set of horizontal edges of $W_{G^{\infty}}$ and $E_{v}\left(W_{G \infty}\right)$ the set of vertical edges of $W_{G^{\infty}}$. We then have:

$$
\sum_{e \in E_{h}\left(W_{G} \infty\right)} \ell(e) \equiv z-x \equiv(2 p+1) \tau_{x}\left(W_{P}\right) \equiv \tau_{x}\left(W_{P}\right) \quad(\bmod 2),
$$

and

$$
\sum_{e \in E_{h}\left(W_{G}\right)} \ell(e) \equiv \sum_{e \in E_{h}\left(W_{G} \infty\right)} 1 \equiv\left|E_{h}\left(W_{G^{\infty}}\right)\right| \quad(\bmod 2) .
$$

Similarly,

$$
\left|E_{v}\left(W_{G^{\infty}}\right)\right| \equiv t-y \equiv q \tau_{y}\left(W_{P}\right) \quad(\bmod 2) .
$$

As $W_{P}$ and $W_{G^{\infty}}$ are of even length, we get:

$$
0 \equiv\left|E\left(W_{G^{\infty}}\right)\right| \equiv q \tau_{y}\left(W_{P}\right)+\tau_{x}\left(W_{P}\right) \quad(\bmod 2) .
$$

\subsection{Regions induced by a coloring of $(P, \pi)$}

The aim of this section is to define a suitable set of walks in order to apply Proposition 37. For this, we will introduce several topological notions.

Definition 38. Let $P_{A B}=P\left[\varphi^{-1}\{a, b\}\right]$ and $P_{C D}=P\left[\varphi^{-1}\{c, d\}\right]$, the subgraphs of $P$ induced by the vertices colored $a$ and $b$ and by the vertices colored $c$ and $d$, respectively. A region $X$ of $P$ is a connected component of $P_{A B}$ or $P_{C D}$. We say that $X$ is of type $a b$ in the first case and of type $c d$ in the latter. The boundary $\partial X$ of a region $X$ is the subset of vertices of $X$ that are adjacent to a vertex not in $X$ :

$$
\partial X=\{x \in X \mid N(x) \nsubseteq X\}
$$

Claim 39. The configuration of Figure 9 cannot appear in the coloring of $\left(P, \pi^{\prime}\right)$. That is to say, for a region $X$ there do not exist two vertices $x, y \in X$ and $w, z \notin X$ such that $x y$, $x z$, yw and $w z$ belong to $E(P)$. We call this configuration the flat border configuration. 


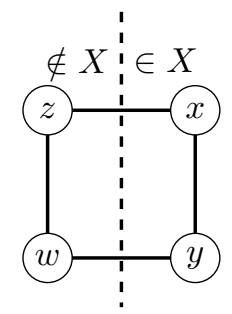

Figure 9: The flat border configuration when $x, y \in X$ and $z, w \notin X$, where $X$ is a region.

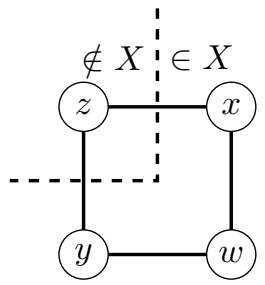

Figure 10: The vertices $x$ and $y$ of the region $X$ are border neighbors.

Proof. Suppose to the contrary that the configuration appears. Then, the cycle $x y w z$ of length 4 is unbalanced. Thus, before switching, $x y w z$ was already an unbalanced cycle of length 4 in $(P, \pi)$ since balance is preserved by switching. By definition of $(P, \pi)$ as a Cartesian product of cycles, the signs of $x y$ and $w z$ are the same. It is also the case for $z x$ and $w y$. Thus this cycle is balanced (it has an even number of negative edges), a contradiction.

Definition 40. Two vertices $x$ and $y$ on the boundary of the region $X$ are border neighbors if $x$ and $y$ have a common neighbor in $X$ and a common neighbor in $P \backslash X$ (see Figure 10). We note $B N(x)$ the set of border neighbors of $x$.

$A$ border $B$ of a region $X$ is a subset of $\partial X$ corresponding to an equivalence class for the transitive closure of the border neighborhood relation (see Figure 11). That is to say, two vertices $x$ and $y$ of $\partial X$ are in the same border $B$ of $X$ if and only if there exists a sequence $u_{0}, u_{1}, \ldots, u_{k}$ of vertices of $B$ such that $u_{0}=x, u_{k}=y$ and for all $0 \leq i<k$, $u_{i}$ and $u_{i+1}$ are border neighbors.

Claim 41. All vertices of a border $B$ of a region $X$ have the same color called the color of $B$.

Proof. By definition of $B$ it suffices to show that any two border neighbors $x$ and $y$ have the same color. Let $z$ be their common neighbor in $X$. Without loss of generality, suppose $X$ is of type $a b$ and $z$ has color $b$. Since the coloring is proper, $x$ and $y$ have color $a$.

Claim 42. A vertex $x$ of a border $B$ has an even number of border neighbors. Moreover if $B N(x)=\varnothing$, then $X=\{x\}$. 


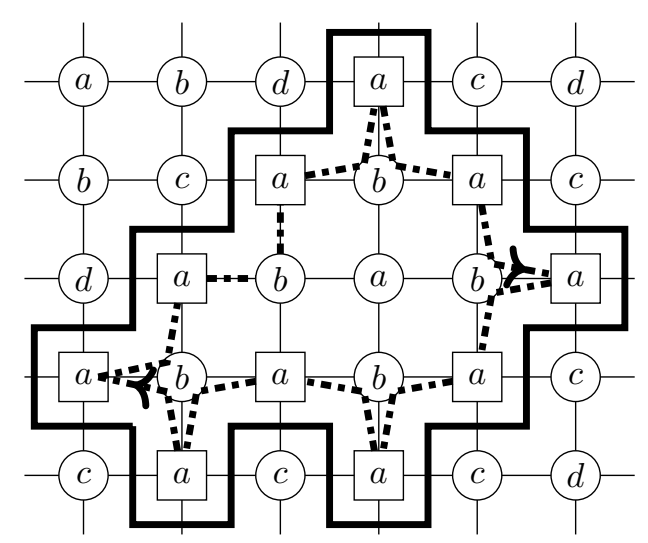

Figure 11: A region $X$ delimited by the bold line and the only border $B$ of $X$ is represented by the square vertices. The dotted line represents the only walk in $\mathcal{W}_{B}$.

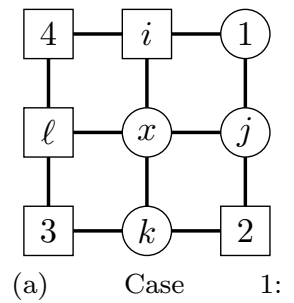

$|B N(x)|=1$.

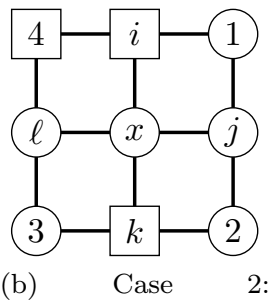

$|B N(x)|=3$.

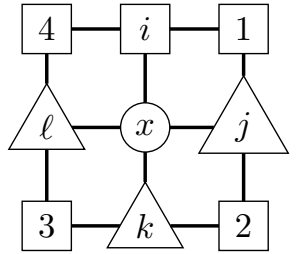

(c) Case: $|B N(x)|=0$.

Figure 12: The two cases up to symmetry where $|B N(x)|$ has an odd number of vertices and the case where $B N(x)=\emptyset$. The square vertices represent vertices not in the region of $x$, the circular ones are in the region while the triangular ones are undecided.

Proof. If $|B N(x)|$ is odd, then we are in one of the first two cases of Figure 12. We will use the notation of the figure.

If $|B N(x)|=1$, then up to rotation and symmetry, we can suppose that the vertex 1 is the border neighbor of $x$ and that $j$ is their common neighbor in $X$. Thus $i \notin X$. Now $\ell \notin X$, as otherwise the vertices $i, \ell, 4$ and $x$ would be in the flat border configuration, which cannot be by Claim 39. The same argument implies $k \in X$ by considering $x, k, j$ and 2. Thus $x, \ell, k$ and 3 are in the flat border configuration. A contradiction.

If $|B N(x)|=3$, then up to rotation and symmetry, we can suppose that the vertex 4 is not a border neighbor of $x$. As 2 is a border neighbor of $x$, one of $k$ and $j$ is in $X$ and the other is not. Without loss of generality, suppose $k \notin X$ and $j \in X$. As 3 is a border neighbor of $x$, we have $\ell \in X$. As 1 is a border neighbor of $x$, we have $i \notin X$. Thus $4, i$, $\ell$ and $x$ are in the flat border configuration, a contradiction.

Now if $B N(x)=\varnothing$, we can suppose that $i \notin X$ as $x$ is in $\partial X$. Now to avoid the flat border configuration, $j, k$ and $\ell$ must not be in $X$. This proves that $X=\{x\}$.

We can now define the set of walks associated with the border.

Definition 43. We associate with a border $B$ of $X$, a set of closed walks $\mathcal{W}_{B}$ in $\left(P, \pi^{\prime}\right)$ included in $X$ (see Figure 11). This set of walks delimits the border of $X$. We use $v_{i, j}$ 


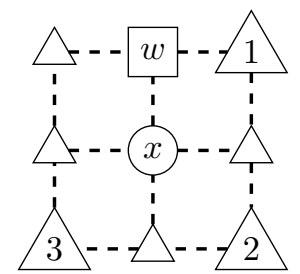

(a) The order of the vertices to choose.

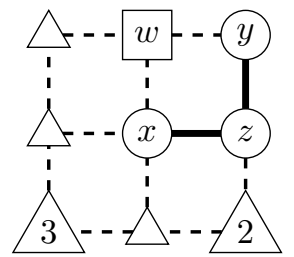

(b) Case 1 .

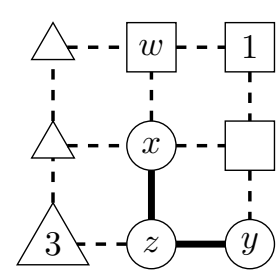

(c) Case 2 .

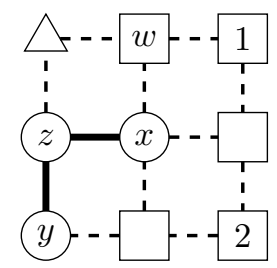

(d) Case 3 .

Figure 13: The first step in constructing the walk. The square vertices represent vertices not in the region of $x$, the circular ones are in the region while the triangular ones are undecided. The dashed edges are the edges of $G^{\infty}$ while the bold edges are the edges of the walk.

to refer to the vertex $\overline{v_{i, j}}$ of $P$ for concision.

We will define the walks piece by piece. In the particular case that $X$ has only one vertex, then $\mathcal{W}_{B}=\varnothing$. Now we can suppose that for each $x \in B$, we have $B N(x) \neq \varnothing$ by Claim 42 .

First pick an arbitrary vertex $x$ of $B$. The vertex $x$ is a border vertex thus there exists at least one vertex $w$ adjacent to $x$ which is not in $X$. In case there are more than one such vertex, we choose one of them arbitrarily. Up to rotation of the coordinate system, we can suppose $x=v_{i, j}$ and $w=v_{i, j+1}$. We will choose $y \in B N(x)$ according to the order in Figure 13a. Meaning the first vertex among $v_{i+1, j+1}, v_{i+1, j-1}$ and $v_{i-1, j-1}$ that belongs to $B N(x)$. The three cases are depicted in Figure 13b, 13c and 13d. Note that as $B N(x)$ is non-empty, $B N(x)$ has at least two vertices by Claim 42 , thus we are in at least one of the three cases above. Through the construction, the "turn left" property, which implies that the vectors $\overrightarrow{s_{2 i} s_{2 i+1}}$ and $\overrightarrow{s_{2 i+1} s_{2 i+2}}$ form a direct base, will be conserved.

Now that we have $x$ and $y$ we can start to construct our walk $W$, by taking $s_{0}=x$, $s_{1}=z$ and $s_{2}=y$ where $z$ is the common neighbor of $x$ and $y$ which is in $X$.

Suppose now that we have constructed the walk up to $s_{0}, \ldots, s_{\ell-2}, s_{\ell-1}, s_{\ell}$ with $\ell$ even. If $s_{\ell-2}=s_{0}, s_{\ell-1}=s_{1}$ and $s_{\ell}=s_{2}$, then we stop and close this walk by removing the last two vertices. Otherwise we will construct $s_{\ell+1}$ and $s_{\ell+2}$. Suppose that $s_{l}=v_{i, j}$. $\mathrm{Up}$ to rotation of the coordinate system, we can suppose that $s_{\ell-2}=v_{i-1, j+1}$ and $s_{\ell-1}=v_{i-1, j}$. The vertex $s_{\ell-1}$ could in principle be $v_{i, j+1}$ but this would contradict the "turn left" property. We construct $s_{\ell+2}$ as the first vertex among $v_{i+1, j+1}, v_{i+1, j-1}$ and $v_{i-1, j-1}$ that belong to $B N(x)$ (see Figure 14a). The three cases are depicted in Figure $14 \mathrm{~b}, 14 \mathrm{c}$ and $14 \mathrm{~d}$. As before, since $B N(x)$ is non empty and of even cardinality, we are in one of those three cases. As in the first step, the vertex $s_{\ell+1}$ is the common neighbor of $s_{\ell}$ and $s_{\ell+2}$ in $X$.

If we stop and there are pairs of border neighbors that are not in the same walk, we 


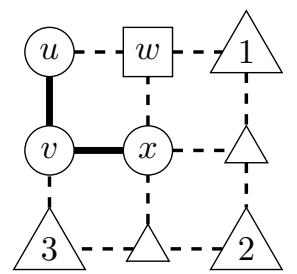

(a) The order of the vertices to choose.

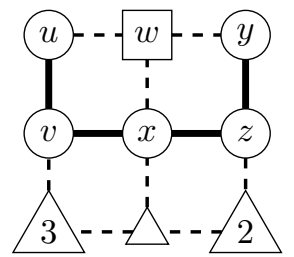

(b) Case 1 .

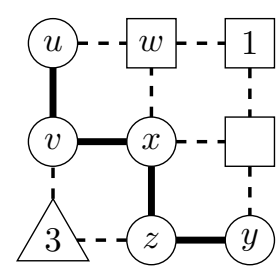

(c) Case 2 .

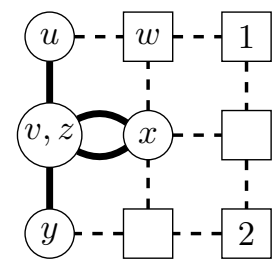

(d) Case 3 .

Figure 14: The next step in constructing the walk. We use the same notation as in Figure 13. We constructed $s_{l-2}=u, s_{l-1}=v, s_{l}=x$. We then construct $s_{l+1}=z$ and $s_{l+2}=y$.

can start the process again with this pair of vertices as the first and third vertices of the walk. To keep the assumption of the construction true, we must carefully choose the start vertex among these two in such a way that the "turn left" property is conserved.

Claim 44. The construction of Definition 43 has the following properties:

1. the construction terminates and all walks are closed,

2. the walks are of even length,

3. all vertices with even indices have the same color,

4. all vertices with odd indices have the same color which is different from the color of the vertices with even indices,

5. all vertices of the border $B$ are vertices of some walk with even index,

6. the number of occurrences of a vertex $x$ of the border $B$ in all the walks of $\mathcal{W}_{B}$ is given by $|B N(x)| / 2$.

Proof. Suppose we do not terminate. As the number of possible edges is finite, the sequence we construct is ultimately periodic. Since $s_{0}, s_{1}, s_{2}$ do not appear consecutively in this order in the rest of the sequence, as we did not stop, the sequence is not periodic. Thus there exists a first moment at which there exist $i$ and $j$ such that $s_{i-2}, s_{i-1}, s_{i}, s_{i+1}, s_{i+2}$ and $s_{j-2}, s_{j-1}, s_{j}, s_{j+1}, s_{j+2}$ are subsequences of the sequence we constructed, verifying $s_{i-2} \neq s_{j-2}, s_{i-1} \neq s_{j-1}, s_{i}=s_{j}, s_{i+1}=s_{j+1}$ and $s_{i+2}=s_{j+2}$. Note that knowing $s_{i-2}, s_{i}$ and $s_{i+2}$ imposes the choices of $s_{i-1}$ and $s_{i+1}$ by the "turn left" property, this is why these two indices exist. Without loss of generality, up to rotating the grid, we can assume that $s_{i}=v_{x, y}$ and $s_{i+2}=v_{x+1, y+1}$. By reversing the construction, we can observe that $s_{i-2}$ is the first border neighbor of $s_{i}$ among $v_{x-1, y+1}$, $v_{x-1, y-1}$ and $v_{x+1, y-1}$ in this order. In this case, $s_{j+2}$ and $s_{i-2}$ are uniquely determined by construction and are equal, a contradiction. Now the process terminates, thus the walks are closed by definition of the terminating condition. This proves 1 . 
Since the walks are included in $X$ which is bipartite, they have even length which proves 2 . In a similar way, all vertices with even indices are on the border $B$ of $X$ thus they have the same color by Claim 41, thus 3 is true. Thus all vertices with odd indices have the other color of $X$ which proves 4 .

We already saw that the vertices of even indices are on $B$. Suppose that $x$ is not part of a walk. We removed the case $|B N(x)|=\varnothing$ by not considering those $B$ 's thus there exists $y \in|B N(x)|$. Then $x$ and $y$ are not in the same walk, thus we create a new one with these two vertices, a contradiction. This proves 5 .

Similarly, if the number of occurrences is strictly smaller than $|B N(x)| / 2$, we would have restarted the process in $x$. Now suppose that this number is strictly greater than $|B N(x)| / 2$. Then there exists a pair of border neighbors $x$ and $y$ that belong to two walks (and there is a vertex $z$ in between them in those two walks) by the pigeon hole principle. Since the construction of the walks only use the position of three consecutive vertices to decide the next two ones, the two walks are identical after passing through $x z y$. By construction, we can choose the start of the walks arbitrarily among the vertices of even indices by shifting the indices, thus we can consider that the two walks start by $x z y$. Thus the two walks are identical which cannot be the case as we would not have restarted to create the second walk.

We define the set of closed walks $\mathcal{W}_{a}$ as the union of all closed walks $\mathcal{W}_{B}$ where $B$ is a border with color $a$.

Take $C$ to be a vertical or horizontal cycle of $P$. For the sake of simplicity, we will take $C$ to be the vertical cycle $\left\{\overline{v_{x, y}} \mid x=x_{0}+n \ell, n \in \mathbb{N}\right\}$ where $\ell=2 p+1$ (i.e. a $U C_{2 q}$-layer). All the following definitions can be stated in the other case by symmetry.

Let $W$ be a closed walk in $P$ (resp. a representation of a closed walk of $P$ in $G^{\infty}$ ). We define a positive crossing of $C$ by $W$ in $P$ (resp. $G^{\infty}$ ) as a sub-walk $t_{0}, t_{1}, \ldots, t_{k-1}, t_{k}$ of $W$ (possibly going through the end of $W$ and going back at the beginning) such that $t_{0}=\overline{v_{x_{0}-1, y}}\left(\right.$ resp. $t_{0}=v_{x_{0}-1+n \ell, y}$ for $n \in \mathbb{N}$ ) for some $y, t_{i} \in C$ for $i \in\{1, \ldots, k-1\}$ and $t_{k}=\overline{v_{x_{0}+1, y^{\prime}}}\left(\right.$ resp. $t_{j}=v_{x_{0}+1+n \ell, y^{\prime}}$ for $\left.n \in \mathbb{N}\right)$ for some $y^{\prime}$. The set of positive crossings $\operatorname{Cross}_{P}^{+}(W, C)$ (resp. $\left.\operatorname{Cross}_{G^{\infty}}^{+}(W, C)\right)$ is the set of all positive crossings of $C$ by $W$ in $P\left(\right.$ resp. $\left.G^{\infty}\right)$.

We can similarly define a negative crossing of $C$ by $W$ in $P$ (resp. $G^{\infty}$ ) by a sub-walk $t_{0}, t_{1}, \ldots, t_{k-1}, t_{k}$ of $W$ (possibly going through the end of $W$ and going back at the beginning) such that $t_{0}=\overline{v_{x_{0}+1, y}}$ (resp. $t_{0}=v_{x_{0}+1+n \ell, y}$ for $n \in \mathbb{N}$ ) for some $y, t_{i} \in C$ for $i \in\{1, \ldots, k-1\}$ and $t_{k}=\overline{v_{x_{0}-1, y^{\prime}}}\left(\right.$ resp. $t_{j}=v_{x_{0}-1+n \ell, y^{\prime}}$ for $n \in \mathbb{N}$ ) for some $y^{\prime}$. We note the corresponding set $\operatorname{Cross}_{P}^{-}(W, C)\left(\operatorname{resp.} \operatorname{Cross}_{G^{\infty}}^{-}(W, C)\right)$.

Claim 45. If $W_{G \infty}$ represents $W_{P}$, then

$\left|\operatorname{Cross}_{P}^{+}\left(W_{P}, C\right)\right|=\left|\operatorname{Cross}_{G^{\infty}}^{+}\left(W_{G^{\infty}}, C\right)\right|$ and $\left|\operatorname{Cross}_{P}^{-}\left(W_{P}, C\right)\right|=\left|\operatorname{Cross}_{G^{\infty}}^{-}\left(W_{G^{\infty}}, C\right)\right|$.

Proof. We will only consider positive crossings, the proof for negative crossings is similar. By taking the quotient of a sub-walk of $W_{G^{\infty}}$, we see that each crossing in $G^{\infty}$ is also present in $P$. Thus $\left|\operatorname{Cross}_{P}^{+}\left(W_{P}, C\right)\right| \supseteq\left|\operatorname{Cross}_{G^{\infty}}^{+}\left(W_{G^{\infty}}, C\right)\right|$. Now take a crossing of $C$ by $W_{P}$ in $P$, it is a sub-walk of $W_{P}$. Thus if we take the corresponding sub-walk in $W_{G^{\infty}}$, we get a crossing in $G^{\infty}$. Thus the two sets are equal.

One of our main results is the following proposition. 


\section{Proposition 46.}

$\sum_{W \in \mathcal{W}_{a}}\left|\operatorname{Cross}_{P}^{+}(W, C)\right|+\left|\operatorname{Cross}_{P}^{-}(W, C)\right| \equiv\{u v \in C \mid$ uv has color ac or ad $\} \quad(\bmod 2)$.

Proof. Take a vertex $x$ of $C$ in $\left(P, \pi^{\prime}\right)$ colored $a$. If the region of $x$ is $\{x\}$, then $x$ has two incident edges colored $a c$ or $a d$ and $x$ is not contained in any crossing as it does not belong to a walk in $\mathcal{W}_{a}$ by definition. Thus we can ignore them.

If $x$ has at least one incident edge colored $a c$ or $a d$, then it belongs to some border colored $a$.

Now take a vertex $x$ of color $a$ in some walk $W \in \mathcal{W}_{a}$. Depending on the size of $B N(x)$ there are one or two occurrences of $x$ in $\mathcal{W}_{a}$ by Claim 44. Up to rotation we can suppose that we have $v_{i-1, j+1}, v_{i-1, j}, v_{i, j}=x$ as a sub-walk of $W$. Depending on the orientation of $C$ (vertical or horizontal), for each sub-case, we must consider the two orientations. For one orientation there are four sub-cases: $|B N(x)|=4,|B N(x)|=2$ and we chose $v_{i+1, j+1}$ during the construction, $|B N(x)|=2$ and we chose $v_{i+1, j-1}$ during the construction or $|B N(x)|=2$ and we chose $v_{i-1, j-1}$ during the construction. All the sub-cases are depicted in Figure 15. In each case the number of crossings for the sub-walks considered is equal, modulo 2 , to the number of edges colored $a b$ of $x$ in $C$.

Now note that no vertices of color $b$ in $W \in W_{a}$ has both neighbors in the same layer. Thus a crossing of $C$ by $W$ always contains a vertex colored $a$ of $C$. Thus all crossings are counted in the above case analysis.

Since for each edge colored $a c$ or $a d$, the vertex colored $a$ has a neighbor not in its region, it is on some border and thus we counted those edges in the case analysis or when we treated the case of the region of size one.

Thus the number of edges colored $a c$ or $a d$ in $C$ is equal to the sum of the number of crossings of $C$ by walks in $\mathcal{W}_{a}$ modulo 2 .

Claim 47. The number of edges colored ac or ad in $C$ is equal to the number of edges of $C$ colored ab modulo 2.

Proof. Let us call $E_{a c}$ (resp. $E_{a d}$, resp. $E_{a b}$ ) the set of edges colored $a c$ (resp. $a d$, resp. $a b)$. Since a vertex of color $a$ has two incident edges in $C$, we have:

$$
\begin{aligned}
\left|\left(E_{a c} \cup E_{a d}\right) \cap C\right| & \equiv \sum_{x \in C \text { of color a }}\left|\left(E_{a c} \cup E_{a d}\right) \cap N(x) \cap C\right| \quad(\bmod 2) \\
& \equiv \sum_{x \in C \text { of color a }} \operatorname{deg}_{C}(x)-\left|E_{a b} \cap N(x) \cap C\right| \quad(\bmod 2) \\
& \equiv \sum_{x \in C \text { of color a }} 2-\left|E_{a b} \cap N(x) \cap C\right| \quad(\bmod 2) \\
& \equiv \sum_{x \in C \text { of color a }}\left|E_{a b} \cap N(x) \cap C\right| \quad(\bmod 2) \\
& \equiv\left|E_{a b} \cap C\right| \quad(\bmod 2) .
\end{aligned}
$$




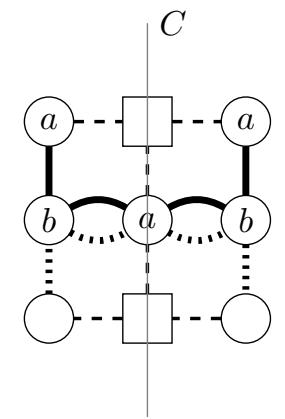

(a) Case 1.a: 2 crossings and 2 edges $a c$ or ad.

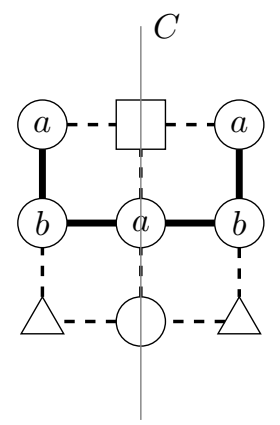

(b) Case 2.a: 1 crossing and 1 edge $a c$ or ad.

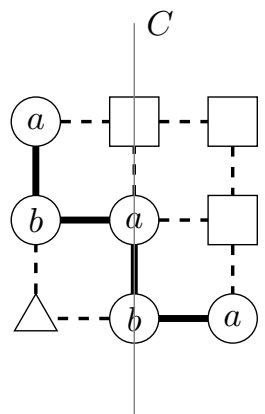

(c) Case 3.a: 1 crossing and 1 edge $a c$ or ad.

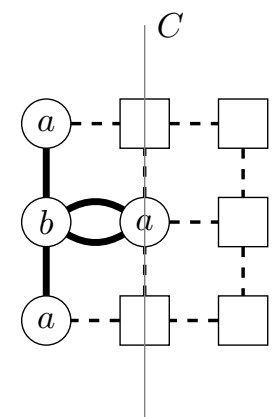

(d) Case 4.a: 0 crossing and 2 edges $a c$ or ad.

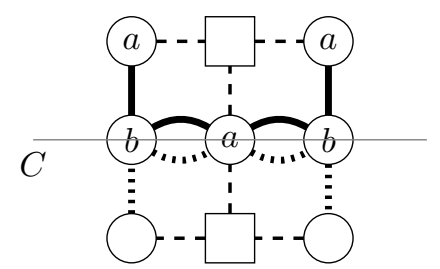

(e) Case 1.b: 0 crossing and 0 edge ac or $a d$.

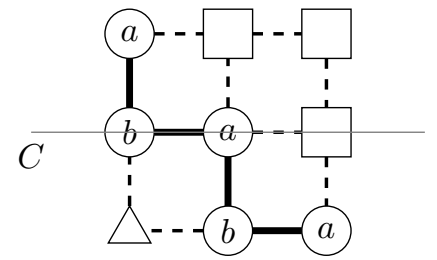

(g) Case 3.b: 1 crossing and 1 edge ac or ad.

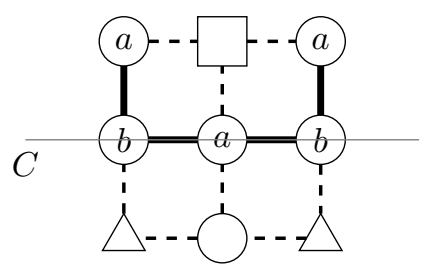

(f) Case 2.b: 0 crossing and 0 edge ac or $a d$.

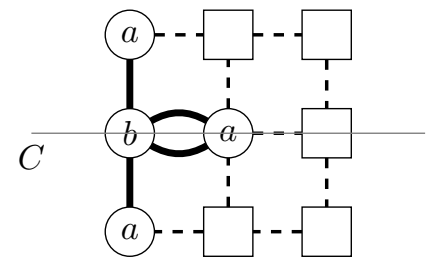

(h) Case 4.b: 1 crossing and 1 edge ac or $a d$.

Figure 15: All cases for the central vertex to belong to a closed walk $W \in \mathcal{W}_{a}$. We use the same notation as in Figure 13. The dotted lines in sub-figures $15 \mathrm{a}$ and $15 \mathrm{e}$ are a second passage in the central vertex by a walk in $\mathcal{W}_{a}$ (possibly the same as the bold line). The edges ac or ad are the edges between a circular vertex and a square vertex. For each case we count the number of crossings of the drawn walks and the number of edges of color $a c$ or $a d$ incident to the central vertex and belonging to the cycle $C$. 


\subsection{Crossings and turns}

In this section, we will suppose that $C$ is the vertical cycle of $\left(P, \pi^{\prime}\right)$ equal to $\left\{\overline{v_{x, y}} \mid x=x_{0}+n \ell, n \in \mathbb{N}\right\}$ for $\ell=2 p+1$. We identify $C$ on $\left(P, \pi^{\prime}\right)$ and the set $\left\{v_{x, y} \mid \overline{v_{x, y}} \in C\right\}$ of vertices of $G^{\infty}$. All what is defined below also works for a horizontal cycle with $\ell=q$. Here we want to connect the number of crossings of the previous section with the number of turns of Section 6.3.

Definition 48. Let $v_{x, y}$ be a vertex of $G^{\infty}$. We define the function $g_{C}$ as follows:

$$
g_{C}\left(v_{x, y}\right)=\left\lfloor\frac{x-x_{0}}{\ell}\right\rfloor .
$$

For a walk $W_{G^{\infty}}$ in $G^{\infty}$ starting at $s_{0}$ and finishing at $s_{n}$, we define $f_{C}$ as follows:

$$
f_{C}\left(W_{G^{\infty}}\right)=g_{C}\left(s_{n}\right)-g_{C}\left(s_{0}\right) .
$$

Claim 49. For a walk $W_{G^{\infty}}$ of $G^{\infty}$ representing a closed walk $W_{P}$ of $P$ with starting point $v_{x, y} \notin C$ :

$$
f_{C}\left(W_{G^{\infty}}\right) \equiv\left|\operatorname{Cross}_{G^{\infty}}^{+}\left(W_{G^{\infty}}, C\right)\right|-\left|\operatorname{Cross}_{G^{\infty}}^{-}\left(W_{G^{\infty}}, C\right)\right| \quad(\bmod 2) .
$$

Proof. Suppose $W_{G^{\infty}}=\left(s_{i}\right)_{i \in\{0, \ldots, n\}}$, by assumption $s_{0} \notin C$. This ensures that all crossings of $C$ by $W_{G \infty}$ are sub-walks that do not go through the end of $W_{G^{\infty}}$ and go back at the beginning. Take a crossing $t_{0}, \ldots, t_{k}$. We have $g_{C}\left(t_{k}\right)-g_{C}\left(t_{0}\right)=1$ if the crossing is positive and $g_{C}\left(t_{k}\right)-g_{C}\left(t_{0}\right)=-1$ if it is negative.

Now we just have to show that the other sub-walks of $W_{G^{\infty}}$ do not contribute to $f_{C}\left(W_{G^{\infty}}\right)$. We can write $W_{G^{\infty}}=W_{0}, W_{0}^{\text {cross }}, W_{1}, \ldots, W_{k-1}^{\text {cross }}, W_{k}$ for some integer $k$ where each $W_{i}^{\text {cross }}$ is a crossing and the other sub-walks are not. Note that:

$$
f_{C}\left(W_{G^{\infty}}\right)=\sum_{i \in\{0, \ldots, k\}} f_{C}\left(W_{i}\right)+\sum_{i \in\{0, \ldots, k-1\}} f_{C}\left(W_{i}^{\text {cross }}\right) .
$$

If for all $i \in\{0, \ldots, k\}, f_{C}\left(W_{i}\right)=0$, we have our result. Since the endpoints of the $W_{i}$ 's are the same as the starting points of the crossings, we know that they do not belong to $C$. The same is true for the starting points. Then, for the starting point $v_{x, y}$ and the endpoint $v_{z, t}$, we have $x, z \in\left\{x_{0}+n \ell+1, \ldots, x_{0}+n \ell+l-1\right\}$ for some $n$. But in all cases the value of $g_{C}$ is $n$. Thus $f_{C}\left(W_{i}\right)=0$. This concludes the proof.

Claim 50. For a closed walk $W_{P}$ in $P$ and $W_{G^{\infty}}$ a representation of $W_{P}$ on $G^{\infty}$ :

$$
f_{C}\left(W_{G \infty}\right) \equiv \tau_{x}\left(W_{P}\right) \quad(\bmod 2) .
$$

Proof. Suppose that $W_{G^{\infty}}$ starts at $v_{x, y}$ and ends at $v_{z, t}$. Note that $z=x+n \ell$ for some $n \in \mathbb{Z}$. We have:

$$
\begin{aligned}
\tau_{x}\left(W_{P}\right) & \equiv\left|\frac{z-x}{\ell}\right| \quad(\bmod 2) \\
& \equiv n \quad(\bmod 2)
\end{aligned}
$$


while:

$$
\begin{aligned}
f_{C}\left(W_{G^{\infty}}\right) & \equiv\left\lfloor\frac{z-x_{0}}{\ell}\right\rfloor-\left\lfloor\frac{x-x_{0}}{\ell}\right\rfloor \quad(\bmod 2) \\
& \equiv n+\left\lfloor\frac{x-x_{0}}{\ell}\right\rfloor-\left\lfloor\frac{x-x_{0}}{\ell}\right\rfloor \quad(\bmod 2) \\
& \equiv n \quad(\bmod 2) .
\end{aligned}
$$

\subsection{End of the proof}

We can now prove Lemma 28.

Proof. Note that by shifting the indices, we can suppose that the starting vertex of each $W_{P} \in \mathcal{W}_{a}$ does not belong to $C$. By using Claim 47, Proposition 46, Claim 45, Claim 49 and Claim 50, in this order, we get:

$$
\begin{aligned}
\left|E_{a b} \cap C\right| & \equiv \sum_{W_{P} \in \mathcal{W}_{a}}\left|\operatorname{Cross}_{P}^{+}\left(W_{P}, C\right)\right|+\left|\operatorname{Cross}_{P}^{-}\left(W_{P}, C\right)\right| \quad(\bmod 2) \\
& \equiv \sum_{\substack{W_{P} \in \mathcal{W}_{a} \\
W_{G \infty} \text { represents } W_{P} \text { and } \\
\text { its starting point } \notin C}}\left|\operatorname{Cross}_{G^{\infty}}^{+}\left(W_{G^{\infty}}, C\right)\right|-\left|\operatorname{Cross}_{G^{\infty}}^{-}\left(W_{G^{\infty}}, C\right)\right| \quad(\bmod 2) \\
& \equiv \sum_{\substack{W_{P} \in \mathcal{W}_{a} \\
W_{G \infty} \text { represents } W_{P} \text { and } \\
\text { its starting point } \notin C}} f_{C}\left(W_{G^{\infty}}\right) \quad(\bmod 2) \\
& \equiv \sum_{W_{P} \in \mathcal{W}_{a}} \tau_{x}\left(W_{P}\right) \quad(\bmod 2) .
\end{aligned}
$$

By the choice of $C$ in the previous subsection, $C=U C_{q}$ and thus $\left|E_{a b} \cap C\right| \equiv 1$ $(\bmod 2)$. Therefore:

$$
1 \equiv \sum_{W_{P} \in \mathcal{W}_{a}} \tau_{x}\left(W_{P}\right) \quad(\bmod 2) .
$$

By taking $C=B C_{2 p+1}$, a horizontal cycle, we obtain:

$$
0 \equiv\left|E_{a b} \cap C\right| \equiv \sum_{W \in \mathcal{W}_{a}} \tau_{y}(W) \quad(\bmod 2) .
$$

Recall that Proposition 37 states that:

$$
0 \equiv q \tau_{y}(W)+\tau_{x}(W) \quad(\bmod 2) .
$$

Thus:

$$
0 \equiv q \times 0+1 \quad(\bmod 2) .
$$

This is a contradiction.

This concludes the proof of Lemma 28. 


\section{Conclusion}

To conclude, in this paper, we showed a number of results on Cartesian products of signed graphs. We proved some algebraic properties: Theorem 8, Theorem 15 and Theorem 17. We also presented an optimal algorithm to decompose a signed graph into its factors in time $O(m)$.

Finally, we computed the chromatic number of Cartesian products: Cartesian products of any graph by a signed forest, Cartesian products of signed paths, signed graphs with underlying graph $P_{n} \square P_{m}$, Cartesian products of some signed complete graphs and Cartesian products of signed cycles. We also presented a tool called an $s$-redundant set that helped to compute chromatic numbers of signed graphs. It would be interesting to determine the exact chromatic number of a signed grid. In this paper, we only presented an upper bound and the question whether 5 or 6 is the best upper bound is still open. It would also be interesting to compute the chromatic number of more Cartesian products.

\section{Acknowledgements}

We would like to thank Hervé Hocquard and Éric Sopena for their helpful comments through the making of this paper. We would also like to thank the reviewers of our submission to CALDAM 2020 for their comments, especially Reviewer 2 of our submission to CALDAM 2020 for pointing us to the techniques of [12] which improved our algorithm. This work is partially supported by the ANR project HOSIGRA (ANR-17-CE40-0022) and the IFCAM project "Applications of graph homomorphisms" (MA/IFCAM/18/39).

\section{References}

[1] F. Aurenhammer, J. Hagauer, and W. Imrich. Cartesian graph factorization at logarithmic cost per edge. computational complexity, 2(4):331-349, Dec 1992.

[2] J.A. Bondy and U.S.R Murty. Graph Theory. Springer Publishing Company, Incorporated, 1st edition, 2008.

[3] C. Duffy, Fabien Jacques, Mickaël Montassier, and Alexandre Pinlou. The chromatic number of 2-edge-colored and signed graphs of bounded maximum degree. ArXiv, abs/2009.05439, 2020.

[4] Tomás Feder. Product graph representations. Journal of Graph Theory, 16(5):467-488, 1992.

[5] Joan Feigenbaum, John Hershberger, and Alejandro A. Schäffer. A polynomial time algorithm for finding the prime factors of cartesian-product graphs. Discrete Applied Mathematics, 12(2):123 $138,1985$.

[6] Antonio Fernández, Tom Leighton, and José Luis López-Presa. Containment properties of product and power graphs. Discrete Applied Mathematics, 155(3):300 - 311, 2007.

[7] K.A. Germina, Shahul Hameed K, and Thomas Zaslavsky. On products and line graphs of signed graphs, their eigenvalues and energy. Linear Algebra and its Applications, 435(10):2432 - 2450 , 2011. Special Issue in Honor of Dragos Cvetkovic.

[8] Frank Harary. On the notion of balance of a signed graph. Michigan Math. J., 2(2):143-146, 1953.

[9] Wilfried Imrich and S Klavžar. Product Graphs, Structure and Recognition. 012000.

[10] Wilfried Imrich, Sandi Klavžar, and Douglas F. Rall. Cancellation properties of products of graphs. Discrete Applied Mathematics, 155(17):2362 - 2364, 2007.

[11] Wilfried Imrich and Iztok Peterin. Recognizing cartesian products in linear time. Discrete Mathematics, 307(3):472 - 483, 2007. Algebraic and Topological Methods in Graph Theory.

[12] Wilfried Imrich and Iztok Peterin. Cartesian products of directed graphs with loops. Discrete Mathematics, 341(5):1336 - 1343, 2018

[13] Reza Naserasr, Edita Rollová, and Éric Sopena. Homomorphisms of signed graphs. Journal of Graph Theory, 79(3):178-212, 2015. 
[14] Gert Sabidussi. Graphs with given group and given graph theoretical properties. Canad. J. Math, 9:515-525, 011957.

[15] Gert Sabidussi. Graph multiplication. Mathematische Zeitschrift, 72:446-457, 1959/60.

[16] V.G. Vizing. The cartesian product of graphs (russian). Vycisl. Sistemy, 9:30-43, 1963.

[17] Peter Winkler. Factoring a graph in polynomial time. European Journal of Combinatorics, 8(2):209 $-212,1987$.

[18] Thomas Zaslavsky. Signed graphs. Discrete Applied Mathematics, 4(1):47 - 74, 1982. 